\title{
Modeling Multi-Cell IEEE 802.11 WLANs with Application to Channel Assignment
}

\author{
Manoj K. Panda and Anurag Kumar \\ Department of Electrical Communication Engineering \\ Indian Institute of Science, Bangalore - 560012. \\ Email: \{manoj,anurag\}@ece.iisc.ernet.in
}

\begin{abstract}
We provide a simple and accurate analytical model for infrastructure IEEE 802.11 WLANs. Our model applies if the cell radius, $R$, is much smaller than the distance, $R_{c s}$, up to which carrier sensing is effective. The condition $R_{c s}>>R$ is likely to hold in a dense deployment of Access Points (APs) where, for every client or station (STA), there is an AP very close to the STA such that the STA can associate with the AP at a high Physical (PHY) rate. We develop a scalable cell level model for such WLANs with saturated AP and STA queues as well as for TCP-controlled long file transfers. The accuracy of our model is demonstrated by comparison with $n s-2$ simulations. We also demonstrate how our analytical model could be applied in conjunction with a Learning Automata (LA) algorithm for optimal channel assignment. Based on the insights provided by our analytical model, we also propose a simple decentralized algorithm which provides static channel assignments that are Nash equilibria in pure strategies for the objective of maximizing normalized network throughput in as many steps as there are channels. In contrast to prior work, our approach to channel assignment is based on the throughput metric.
\end{abstract}

Index Terms - throughput modeling, fixed point analysis, channel assignment algorithm, Nash equilibria, learning automata

\section{INTRODUCTION}

This paper is concerned with infrastructure mode Wireless Local Area Networks (WLANs) that use the Distributed Coordination Function (DCF) Medium Access Control (MAC) protocol as defined in the IEEE 802.11 standard [1]. Such WLANs contain a number of Access Points (APs). Each client station (STA) associates with exactly one AP. Each AP, along with its associated STAs, defines a cell. Each cell operates on a specific channel. Cells that operate on the same channel are called co-channel. Thus, in our setting, DCF is used only for single-hop communication within the cells, and the STAs can access the Internet only through their respective APs, which are connected to the Internet by a high-speed wireline local area network. Figure-1 depicts a multi-cell infrastructure WLAN.

To support the ever-increasing user population at high access speeds, WLANs are resorting to dense deployments of APs where, for every STA, there exists an AP close to the STA with which the STA can associate at a high Physical (PHY) rate [2]. However, as the density of APs increases, cell sizes become smaller and, since the number of non-overlapping channels is limited 1 , co-channel cells become closer. Nodes

\footnotetext{
${ }^{1}$ For example, the number of non-overlapping channels in $802.11 \mathrm{~b} / \mathrm{g}$ is 3 and that in $802.11 \mathrm{a}$ is 12 .
}

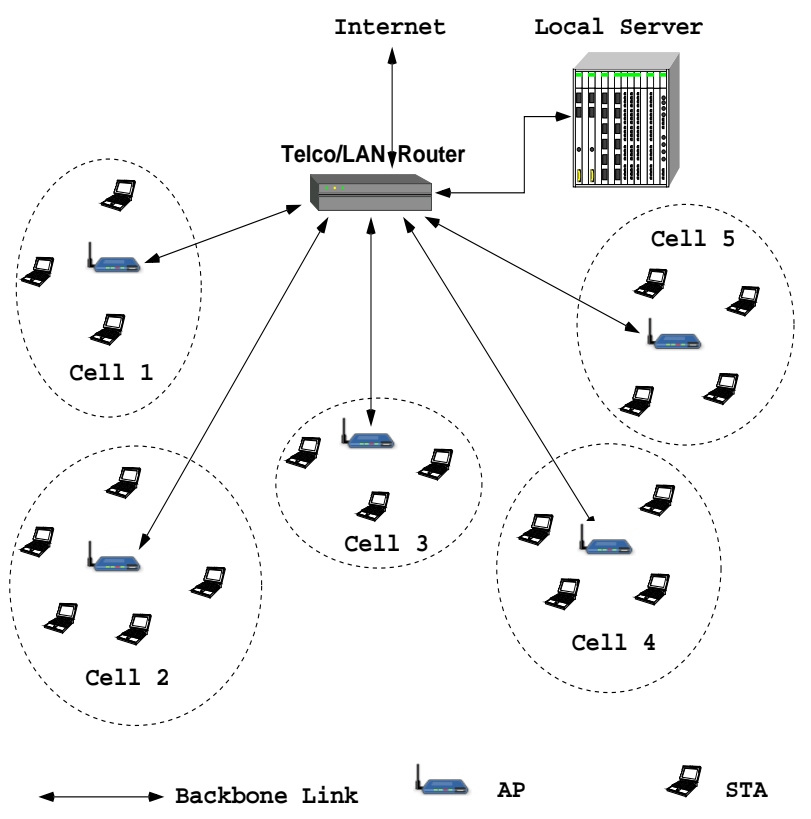

Fig. 1. A multi-cell infrastructure WLAN: DCF is used only for communications within the cells. Connectivity to nodes outside the cell is provided over a separate backbone which connects the APs to the Internet through a local server and a LAN router.

in two closely located co-channel cells can suppress each other's transmissions via carrier sensing and interfere with each other's receptions causing packet losses. Thus, capacity can sometimes degrade with increased AP density [3], [4]. Clearly, effective planning and management are essential for achieving the benefits of dense deployments of APs [2]. It has been demonstrated that a dense deployment of APs, along with careful channel assignment and user association control, can enhance the capacity by as much as $800 \%$ [2]; but the technique has been tested on a small scale 2 . Large-scale WLANs are difficult to plan and manage since good network engineering models are lacking.

Much of the earlier work on modeling WLANs deals with single-AP networks or the so-called single cells [5], [6], [7]. The existing performance analyses of multi-cell WLANs (i.e., WLANs consisting of multiple APs) are mostly based either on simulations or small-/medium-scale experiments

\footnotetext{
${ }^{2}$ In [2], to provide connectivity at high PHY rates, 24 APs were deployed in an area which could have been covered by a single AP at a low PHY rate.
} 
[3], [4], [2]. Studying large-scale WLANs by simulations and experiments is both expensive and time-consuming. It is, therefore, important to develop an analytical understanding of such WLANs in order to derive insights into the system dynamics. The insights thus obtained can be applied: (i) to facilitate planning and management, and (ii) to develop efficient adaptive schemes for making the WLANs selforganizing and self-managing. In this paper, we first develop an analytical model for 802.11-based multi-cell WLANs and then apply our model to the task of channel assignment.

Contributions: We make the following contributions:

- We identify a condition, which we call the Pairwise Binary Dependence (PBD) condition, under which multicell WLANs can be modeled at the cell level (see A4 in Section IV].

- We develop a scalable cell level model for multi-cell WLANs with arbitrary cell topologies (Section $\mathrm{V}$ ).

- We extend the single cell TCP analysis of [8] to multiple interfering cells (Section $\mathrm{V}-\mathrm{B}$ ).

- We demonstrate how our model could be applied in conjunction with a Learning Automata (LA) algorithm for optimal channel assignment (Section (VIII).

- Based on the insights provided by our analytical model, we propose a simple decentralized algorithm which can provide fast static channel assignments (Section IX).

Unlike the node level models [9], [10] or the link level models [11], [12] reported earlier in the context of multihop ad hoc networks, our cell level model does not require modeling the activities of every single node (resp. link). Thus, the complexity of our cell level model increases with the number of cells rather than the number of nodes (resp. links). However, our model can account for the number of nodes in each cell which may differ across the cells. We argue that the PBD condition is likely to hold in a dense deployments of APs (see Sections III] and IV-A). Hence, our cell level model can be applied to obtain a first-cut understanding of large-scale WLANs.

Our cell level model is based on the channel contention model of Boorstyn et al. [9] and the transmission attempt model of [7]. Thus, our approach is similar to that of [10]. However, we provide a cleaner treatment by modifying the model of [9] to make it suitable for 802.11 networks (see the discussion following Equation 22. We also provide new insights.

Our channel assignment algorithm provides assignments that are Nash equilibria in pure strategies for the normalized network throughput maximization objective (see Section [X]). Furthermore it provides an assignment in only $M-1$ steps where $M$ denotes the number of available channels. In contrast to prior work, our approach to channel assignment is based on the throughput metric (see the last paragraph in Section II).

The remainder of this paper is organized as follows. In Section [II we discuss the related literature. In Section [III we provide the motivation for our simple cell level model. In
Section IV we provide our network model and summarize our key modeling assumptions. The analytical model is developed in Section V In Section VI we validate our model by comparing with $n s-2$ simulations. In Section VII we apply our model to compare three different channel assignments for a 12-cell network and discuss how our model can be applied for optimal channel assignment in small networks using exhaustive search. In Section VIII, we demonstrate how our analytical model could be applied along with Learning Automata (LA) algorithms for optimal channel assignment. We propose a decentralized algorithm in Section [IX which quickly provides static channel assignments. We conclude in Section $\mathrm{X}$ Proofs of important results can be found in the appendices at the end of the paper.

\section{RELATED LitERATURE}

Modeling of multi-hop ad hoc networks is closely related to that of multi-cell WLANs. In both cases, the hidden node and the exposed node problems [13] are known to be the two main capacity-degrading factors. Multi-hop ad hoc networks and multi-cell WLANs are much harder to model than single cells precisely because of the presence of hidden and exposed nodes. In particular, since each node evolves in a different specific way, one needs to model the system at the node level [9], [10], or at the link level [11], [12], i.e., the activities of every single node or link and the interactions among them need to be modeled.

In the context of CSMA based multi-hop packet radio networks, Boorstyn et al. [9] proposed a Markovian model with Poisson packet arrivals and arbitrary packet length distributions. Wang and Kar [11] adopted the node level Boorstyn model to develop a link level model for 802.11 networks and studied the fairness issues. As a simplification, they assumed a fixed contention window. Garetto et al. [10] extended the Boorstyn model to multi-hop 802.11 networks. They computed the packet loss probabilities and the throughputs per node accounting for the details of the 802.11 protocol. In particular, they incorporated the evolution of contention windows, which was missing in [11], by applying the analysis of [7]. However, a direct application of the Boorstyn model required two different activation rates, namely, $\lambda$ and $g$, which complicated their model (see the discussion following Equation 2). We develop a cleaner model by slightly modifying the Boorstyn contention model and making it particularly suitable for 802.11 networks.

In the context of multi-cell infrastructure WLANs, Nguyen et al. [14] proposed a model for dense 802.11 networks. To keep their interference analysis tractable, they assumed all the APs in the WLAN to be operating on the same channel. Recently, Bonald et al. [15] applied the concept of "exclusion region" to model a multi-AP WLAN as a network of multiclass processor-sharing queues with state-dependent service rates. The concept of "exclusion domains" has also been applied in [12] to study the long-term fairness properties of large networks. The concept of exclusion says that, among a set of neighboring links, at most one can be active at any 
point of time. As argued in [12] and [15], exclusion can be enforced in 802.11 networks by the RTS/CTS mechanism. However, suppression of interferers by RTS/CTS is not perfect because: (1) RTS frames can also collide and they must be retransmitted to enforce exclusion, (2) the overheads due to RTS/CTS and the capacity wastage due to RTS collisions might be significant, especially at high data rates [16], and hence, must be accounted for, and most importantly, (3) RTS/CTS cannot completely eliminate hidden node collisions since nodes that cannot decode the CTS frames may collide with DATA frames that are longer than the Extended Inter Frame Space (EIFS). Thus, "exclusion" is strongly dependent on the RTS/CTS mechanism and is, essentially, a modeling approximation which ignores the possibility of hidden node collisions. We also ignore hidden node collisions to develop our model. However, our model can be applied either with the Basic Access mechanism or with the RTS/CTS mechanism.

Several techniques exist for improving the throughput and fairness in WLANs. 'Channel assignment' is performed at the planning stage and dynamic techniques such as 'power control', 'tuning of carrier sense threshold', 'channel switching' and 'user association control' can be applied during network operation. Extensive literature exists on these techniques (see, e.g., [13], [17], [18], [19], [20], [21], [22], [23] and the references therein). In this paper, we focus only on channel assignment. Much of the existing work on channel assignment proposes to minimize the global interference power or maximize the global Signal to Noise and Interference Ratio (SINR) without taking into account the combined effect of the PHY and the MAC layers. Due to carrier sensing, nodes in 802.11 networks get opportunity to transmit only a fraction of time which must be accounted for when computing the global interference power or SINR. Such an approach is found only in [17] where the authors propose to maximize a quantity called "effective channel utilization". In reality, end users are more interested in the "throughputs" and, ideally, the objective should be to maximize the sum utility of throughputs. To our knowledge, a throughput based approach which accounts for PHY-MAC interaction does not exist. In this paper, we first develop a simple and accurate throughput model for multicell WLANs and then apply our model for the task of channel assignment.

\section{Motivation for a Cell Level Model}

In a dense deployment of APs with denser user population, it seems practically impossible to apply a node or a link level model for planning and managing the network. However, we can exploit a specific characteristics of dense deployments. Let $R$ denote the maximum AP-STA distance and let $R_{c s}$ denote the carrier sensing range, i.e., $R_{c s}$ is the distance up to which carrier sensing is effective 3 . We observe that, $R_{c s}>>R$ is likely to hold in a dense deployment of APs where, for every $\mathrm{STA}$, there is an AP very close to the STA. With $R_{c s}>>R$, the network model can be simplified as follows: (1) since any

\footnotetext{
${ }^{3}$ Precise definition of carrier sensing range can be found in [16] and [13].
}

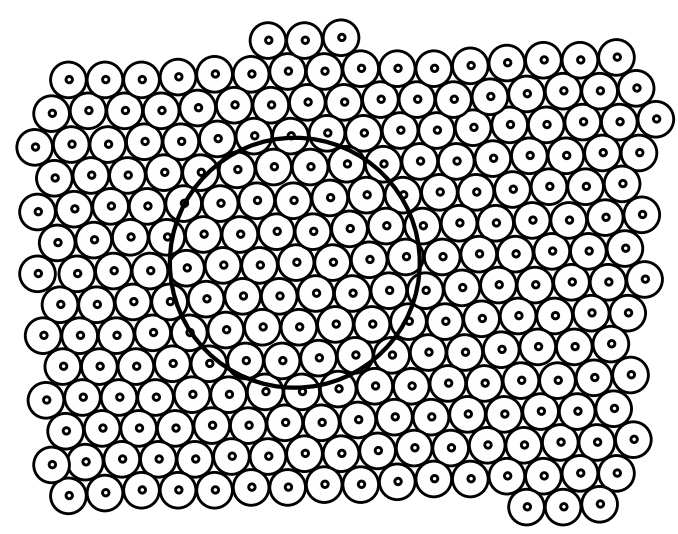

Fig. 2. A dense AP network: Small circular areas represent cells with radius $R$. APs are shown as tiny circles at the centers of the cells. The big circular disk represents the area covered by the carrier sensing range $R_{c s}$ of one of the APs. Observe that, with $R_{c s}>>R$, other cells are either almost completely coved or almost not covered at all through carrier sensing.

transmitter ' $\mathrm{T}$ ' is within a small distance $R$ from its receiver ' $\mathrm{R}$ ', a node 'I' that is beyond a distance $R_{c s}$ from ' $\mathrm{T}$ ' (i.e., a hidden node) is unlikely to interfere with ' $\mathrm{R}$ ', and (2) if Node- 1 in Cell- 1 can sense the transmissions by Node- 2 in Cell-2, then it is likely that all the nodes in Cell-1 can sense the transmissions from all the nodes in Cell- 2 and vice versa, i.e., we may assume that nodes belonging to the same cell have an identical view of the rest of the network and interact with the rest of the network in an identical manner.

The assumption that the AP and all its associated STAs have an identical view of the network has been applied in a dense AP setting [2] where the authors approximate STA statistics by statistics collected at the APs for efficiently managing their network. We adopt this idea of [2] to develop an analytical model. We identify the locations of the STAs with the locations of their respective APs, and treat a cell as a single entity, thus yielding a scalable cell level model. Unlike a node level model, the complexity of a cell level model increases with the number of cells rather than number of nodes. A simple cell level model is particularly suitable for the task of channel assignment since channels are assigned to cells rather than to nodes. The foregoing simplification is extremely useful at the network planning stage when the locations of the STAs are not known but the locations of the APs and the expected number of users per cell might be known. Furthermore, since much of the traffic in today's WLANs is downlink, i.e., from the APs to the STAs, a large fraction of channel time is occupied by transmissions from the APs. It is then reasonable to assign channels based only on the topology of the APs and the expected number of users per cell assuming that the users are located close to their respective APs.

The appropriate locations for placing the APs can be decided using standard RF tools and the physical topology of the APs can be determined. Given the physical topology 
of the APs and a channel assignment, we can obtain the logical topology, i.e., the topology of the co-channel APs corresponding to every channel. Given a logical topology, our analytical model can predict the cell throughputs accounting for the expected number of users per cell. The cell throughputs thus obtained can provide the goodness of the assignment to a channel assignment algorithm based on which the algorithm can determine a better channel assignment. Given the logical topology with the new channel assignment, our model can provide the modified feedback and this procedure can be repeated several times to arrive at an optimal assignment. We explain this approach in Section VIII where we apply our model in conjunction with Learning Automata (LA) based algorithms for optimal channel assignment.

\section{Network Model And Assumptions}

Based on our earlier discussion, we assume that simultaneous transmissions by nodes that are farther than $R_{c s}$ from each other results in successful receptions at their respective receivers. We also assume that simultaneous transmissions by nodes that are within $R_{c s}$ always lead to packet losses at their respective receivers, i.e., we ignore the possibility of packet capture. We say that two nodes are dependent if they are within $R_{c s}$; otherwise, the two nodes are said to be independent. Two cells are said to be independent if every node in a cell is independent w.r.t. every node in the other cell; otherwise, the two cells are said to be dependent. Two dependent cells are said to be completely dependent if every node in a cell is dependent w.r.t. every node in the other cell. In this broad setting, our key assumptions are the following:

A.1 Only non-overlapping channels are used.

A.2 Cells operating on different channels are independent.

A.3 Associations of STAs with APs are static. This implies that the number of STAs in a cell is fixed.

A.4 Pairwise Binary Dependence (PBD): Any pair of cells is either independent or completely dependent.

A.5 The STAs are so close to their respective APs that packet losses due to channel errors are negligible.

A.6 The EIFS deferral has been disabled in the sense that medium access always starts after a Distributed InterFrame Space (DIFS) deferral.

\section{A. Discussion of the PBD Condition and Assumption A, 6}

The PBD condition is crucial to the analytical model being developed in this paper. It includes the possibility that two given co-channel cells can be independent, i.e., two co-channel cells can be so far apart that activities in one cell do not affect the activities in the other cell. However, if two cells are dependent, the PBD condition rules out the possibility that only a subset of nodes in one cell can sense a subset of nodes in the other cell. We note that, in a dense deployment of APs, due to small cell radius $R$ and $R_{c s}>>R$, the PBD condition would hold, at least approximately. The PBD condition is a geometric property that enables modeling at the cell level since, if the PBD condition holds, the relative locations of the nodes within a cell do not matter. Also, any interferer
'I', which must be within $R_{c s}$ of a receiver 'R', will also be within $R_{c s}$ of the transmitter 'T' if the PBD condition holds. Hence, either (i) 'I' can start transmitting at the same time as ' $\mathrm{T}$ ' causing synchronous collisions at 'R', or (ii) ' $\mathrm{I}$ ' gets suppressed by T's RTS (or DATA) transmission followed by R's CTS (or ACK) transmission since CTS and ACK frames are given higher priority through SIFS $(<$ DIFS $<$ EIFS). Thus, if the PBD condition holds, nodes do not require deferral by EIFS; deferral by DIFS would suffice. We assume that contention for medium access always begins after deferral by DIFS and we do not model the impact of EIFS.

\section{Analysis of Multi-Cell WLANs with ARbitrary Cell Topology}

In this section, we develop a cell level model for WLANs that satisfy the PBD condition. We provide a generic model and demonstrate the accuracy of our model by comparing with simulations of specific cell topologies pertaining to: (i) planned networks with linear or hexagonal layout of cells (see Figures 3(a) 3(c) , and (ii) unplanned networks with arbitrary layout of cells (see Figure 3(d)). We index the cells by positive integers $1,2, \cdots, N$, in some arbitrary fashion where $N$ denotes the number of cells. Two completely dependent co-channel cells are said to be neighbours. Note that two completely dependent cells operating on different channels are not neighbors (see $\mathrm{A} 2$ ). Let $\mathcal{N}=\{1,2, \cdots, N\}$ denote the set of cells and $\mathcal{N}_{i}(\subset \mathcal{N})$ denote the set of neighboring cells of Cell- $i(i \in \mathcal{N})$. Note that $i \notin \mathcal{N}_{i}$. The key to modeling the cell level contention is the cell level contention graph $\mathcal{G}$ which is obtained by representing every cell by a vertex and joining every pair of neighbors by an edge. Figures $3(\mathrm{a}) 3(\mathrm{~d})$ also depict the contention graphs corresponding to each cell topology.

In Section $\mathrm{V}-\mathrm{A}$, we model the case where nodes are infinitely backlogged and are transfering packets to one or more nodes in the same cell using UDP connection(s). Notice that single-hop direct communications among the STAs in the same cell without involving the AP are also allowed. In Section V-B, we extend to the case when STAs download long files through their respective APs using persistent TCP connections.

\section{A. Modeling with Saturated MAC Queues}

Due to the PBD condition, nodes belonging to the same cell have an identical view of the rest of the network. When one node senses the medium idle (resp. busy) so do the other nodes in the same cell and we say that a cell is sensing the medium idle (resp. busy). Since the nodes are saturated, whenever a cell senses the medium idle, all the nodes in the cell decrement their back-off counters per idle back-off slot that elapses in their local medium 4 and we say that the cell is in backoff. If the nodes were not saturated, a node with an empty MAC queue would not count down during the "medium idle" periods. We say that a cell transmits when one or more nodes

\footnotetext{
${ }^{4}$ Nodes belonging to different co-channel cells can have different views of the network activity.
} 


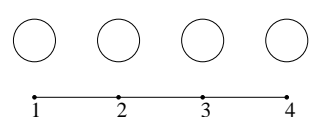

(a)

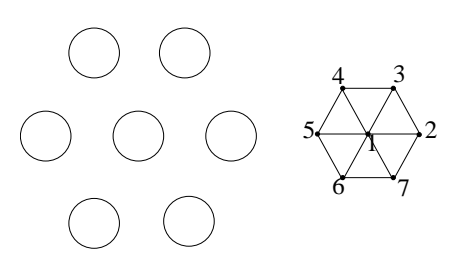

(c)

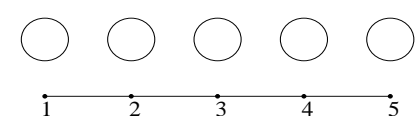

(b)

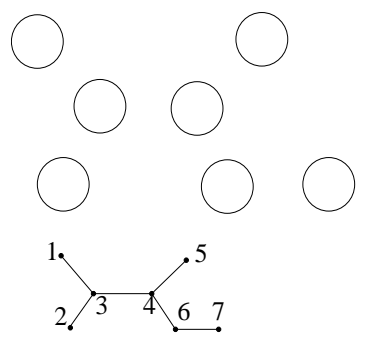

(d)
Fig. 3. Examples of multi-cell systems: (a) four linearly placed co-channel cells, (b) five linearly placed co-channel cells, (c) seven hexagonally placed co-channel cells, and (d) seven co-channel cells with an arbitrary cell topology. The cell level contention graphs have also been shown where the dark dots represent the cells. Neighbors have been joined by edges. For example (a), the pairs $\{1,2\},\{2,3\}$ and $\{3,4\}$ are dependent and the pairs $\{1,3\},\{1,4\}$ and $\{2,4\}$ are independent.

in the cell transmit(s). When two or more nodes in the same cell transmit, an intra-cell collision occurs.

Consider Figure 3(a) There are periods during which all the four cells are in back-off. We model these periods, when none of the cells is transmitting, by the state $\Phi$ where $\Phi$ denotes the empty set. The system remains in State $\Phi$ until one or more cell(s) transmit(s). When a cell transmits, its neighbors sense the transmission after a propagation delay and they defer medium access. We then say that the neighbors are blocked due to carrier sensing. However, two neighboring cells can start transmitting together before they could sense each other's transmissions resulting in synchronous inter-cell collisions. Note that intra-cell collisions are always synchronous and hidden node collisions are mostly asynchronous. Due to the PBD condition, there cannot be hidden node collisions.

We observe that a cell can be in one of the three states: (i) transmitting, (ii) blocked, or (iii) in back-off. Modeling the synchronous inter-cell collisions requires a discrete time slotted model. However, this would require a large state space since the cells change their states in an asynchronous manner. For example, consider Figure 3(a) and suppose that Cell1 starts transmitting and blocks Cell-2 after a propagation delay. However, Cell-3 is independent of Cell-1 and can start transmitting at any instant during Cell-1's transmission. Thus, the evolution of the system is partly asynchronous (since state transitions occur asynchronously) and partly synchronous (since both inter-cell and intra-cell collisions are synchronous). To capture both, we follow a two-stage approach. In the first stage, we ignore inter-cell collisions and assume that blocking due to carrier sensing is immediate. We develop a continuous time model along the lines of [9] to obtain the fraction of time each cell is transmitting/blocked/in back-off. In the second stage, we obtain the fraction of slots in which various subsets of neighboring cells can attempt together. This would allow us to compute the collision probabilities accounting for synchronous inter-cell collisions. We combine the above through a fixed-point equation and compute the throughputs using the solution of the fixed-point equation.

The above two-stage approach is based on [10]. However, our model of transmission attempt process is simpler (see the discussion following Equation 2) and the closed-form expressions for collision probabilities and cell throughputs that we derive are new. We define the following [7]:

$n_{i}:=$ number of nodes in Cell- $i$

$\beta_{i}:=$ (transmission) attempt probability (over the back-off slots) of the nodes in Cell- $i$

$\gamma_{i}:=$ The collision probability as seen by the nodes in Cell $-i$ (conditioned on an attempt being made)

More precisely, let $A_{l}^{(i)}$ and $C_{l}^{(i)}$ denote the cumulative number of attempts and collisions of a node (any node) in Cell$i$ up to the the $l^{\text {th }}$ back-off slot boundary. Then, the attempt probability $\beta_{i}$ and the (conditional) collision probability $\gamma_{i}$ of the node are defined as:

$$
\beta_{i}:=\lim _{l \rightarrow \infty} \frac{A_{l}^{(i)}}{l} ; \gamma_{i}:=\lim _{l \rightarrow \infty} \frac{C_{l}^{(i)}}{A_{l}^{(i)}} .
$$

Note that $\beta_{i}$ is the attempt probability of the nodes in Cell$i$, irrespective of whether the nodes in the other cells can also attempt. This is a simplification and can be viewed as an extension to the decoupling approximation introduced in [5]. Using the decoupling approximation and the analysis in [7], the attempt probability $\beta_{i}$ of the nodes in Cell- $i, \forall i \in \mathcal{N}$, can be related to $\gamma_{i}$ as

$$
\beta_{i}=G\left(\gamma_{i}\right):=\frac{1+\gamma_{i} \cdots+\gamma_{i}^{K}}{b_{0}+\gamma_{i} b_{1} \cdots+\gamma_{i}^{k} b_{k}+\cdots+\gamma_{i}^{K} b_{K}}
$$

where $K$ denotes the retry limit and $b_{k}, 0 \leq k \leq K$, denotes the mean back-off sampled after $k$ collisions.

The First Stage: When Cell- $i$ and some (or all) of its neighboring cells are in back-off their (cell level) attempt processes compete until one of the cells, say, Cell- $j, j \in$ $\mathcal{N}_{i} \cup\{i\}$, transmits. Since, we ignore inter-cell collisions in the first stage, the possibility of two or more neighboring cells attempting together is ruled out. Thus, the first stage of our model is similar to the exclusion model proposed in [15] and [12]. When Cell- $i$ wins the contention, we say that it has become active. When Cell- $i$ becomes active, it gains the control over its local medium by immediately blocking its neighboring cells that are not yet blocked. We assume that the time until Cell- $i$ goes from the back-off state to the active state is exponentially distributed with mean $\frac{1}{\lambda_{i}}$. The activation rate $\lambda_{i}$ is given by

$$
\lambda_{i}=\frac{1-\left(1-\beta_{i}\right)^{n_{i}}}{\sigma}
$$


where $\sigma$ denotes the duration of a back-off slot (in seconds) and $1-\left(1-\beta_{i}\right)^{n_{i}}$ is the probability that there is an attempt in Cell- $i$ per back-off slot. Notice that we have converted the aggregate attempt probability in a cell per back-off slot to an attempt rate over back-off time. Also notice that, our assumption of exponential "time until transition from the backoff state to the active state" is the continuous time analogue of the assumption of geometric "number of slots until attempt" in the discrete time models of [6] and [7].

Discussion 5.1: In [10], the authors use an unconditional activation rate $\lambda$ over all times as well as a conditional activation rate $g$ over the back-off times and relate the two rates through a throughput equation which makes their model unnecessarily complicated. The activation rate $\lambda$ in our model is conditional on being in the back-off state. Thus, we use a single activation rate and our model is much simpler than that of [10]. Our modified approach can also be applied to simplify the node level model of [10].

When Cell- $i$ becomes active, its neighbors remain blocked due to Cell- $i$ until Cell-i's transmission finishes and an idle DIFS period elapses. The active periods of Cell- $i$ are of mean duration $\frac{1}{\mu_{i}}$. When Cell $i$ becomes active through a successful transmission (resp. an intra-cell collision) its neighbors remain blocked due to Cell- $i$ for a success time $T_{s}$ (resp. a collision time $T_{c} \sqrt{5}$. Hence, $\frac{1}{\mu_{i}}$ is given by

$$
\begin{aligned}
\frac{1}{\mu_{i}}= & \left(\frac{n_{i} \beta_{i}\left(1-\beta_{i}\right)^{n_{i}-1}}{1-\left(1-\beta_{i}\right)^{n_{i}}}\right) \cdot\left(T_{s}\right) \\
& +\left(1-\frac{n_{i} \beta_{i}\left(1-\beta_{i}\right)^{n_{i}-1}}{1-\left(1-\beta_{i}\right)^{n_{i}}}\right) \cdot\left(T_{c}\right)
\end{aligned}
$$

where $\frac{n_{i} \beta_{i}\left(1-\beta_{i}\right)^{n_{i}-1}}{1-\left(1-\beta_{i}\right)^{n_{i}}}$ is the probability that Cell- $i$ becomes active through a success given that it becomes active.

Due to carrier sensing, at any point of time, only a set $\mathcal{A}(\subset \mathcal{N})$ of mutually independent cells can be active together, i.e., $\mathcal{A}$ must be an independent set (of vertices) of the contention graph $\mathcal{G}$. From the cell level contention graph we can determine the set of cells $\mathcal{B}_{\mathcal{A}}$ that get blocked due to $\mathcal{A}$, and the set of cells $\mathcal{U}_{\mathcal{A}}$ that remain in back-off. Note that $\mathcal{A}, \mathcal{B}_{\mathcal{A}}$ and $\mathcal{U}_{\mathcal{A}}$ form a partition of $\mathcal{N}$, i.e., $\mathcal{A}, \mathcal{B}_{\mathcal{A}}$ and $\mathcal{U}_{\mathcal{A}}$ are pairwise disjoint and $\mathcal{N}=\mathcal{A} \cup \mathcal{B}_{\mathcal{A}} \cup \mathcal{U}_{\mathcal{A}}$.

We take $\mathcal{A}(t)$, i.e., the set $\mathcal{A}$ of active cells at time $t$, as the state of the multi-cell system at time $t$. It is worthwhile now to mention the insensitivity result of Boorstyn et al. [9] which says that the product-form solution provided by their model is insensitive to the packet length distribution and depends only on the mean packet lengths. Applying their insensitivity argument, we take the active periods of Cell- $i$ to be i.i.d exponential random variables with mean $\frac{1}{\mu_{i}}$. Then, at any time $t$, the next state and the rate of transition to the next state is completely determined by the current state $\mathcal{A}(t)$. For example, Cell- $j, j \in \mathcal{U}_{\mathcal{A}}$, joins the set $\mathcal{A}$ (and its neighboring cells that

\footnotetext{
${ }^{5}$ For the Basic Access (resp. RTS/CTS) mechanism, $T_{s}$ corresponds to the time DATA-SIFS-ACK-DIFS (resp. RTS-SIFS-CTS-SIFS-DATA-SIFS-ACKDIFS) and $T_{c}$ corresponds to the time DATA-DIFS (resp. RTS-DIFS).
}

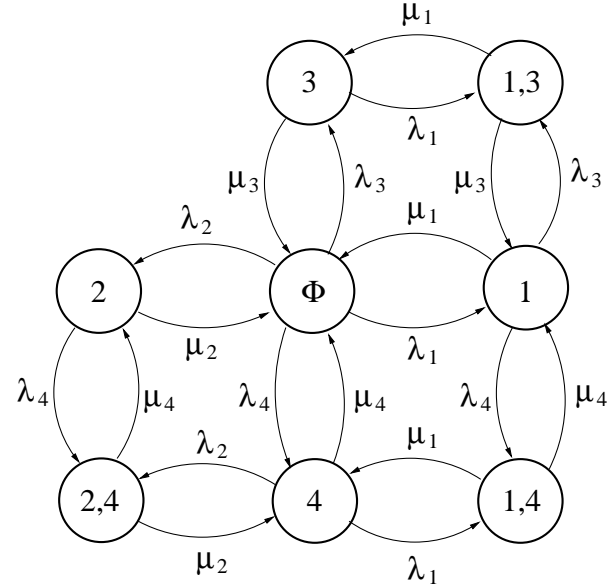

Fig. 4. The CTMC describing the cell level contention for the four linearly placed cells given in Figure 3(a)

are also in $\mathcal{U}_{\mathcal{A}}$ join the set $\mathcal{B}_{\mathcal{A}}$ ) at a rate $\lambda_{j}$. Similarly, Cell- $i$, $i \in \mathcal{A}$, leaves the set $\mathcal{A}$ (and its neighboring cells that are blocked only due to Cell- $i$ leave the set $\mathcal{B}_{\mathcal{A}}$ ) to join the set $\mathcal{U}_{\mathcal{A}}$ at a rate $\mu_{i}$. In summary, the process $\{\mathcal{A}(t), t \geq 0\}$ has the structure of a Continuous Time Markov Chain (CTMC). This CTMC contains a finite number of states and is irreducible. Hence, it is stationary and ergodic.

The set of all possible independent sets which constitutes the state space of the CTMC $\{\mathcal{A}(t), t \geq 0\}$ is denoted by $\mathcal{A}$. For a given contention graph, $\mathcal{A}$ can be determined. For the topology given in Figure 3(a) we have $\mathcal{N}=\{1,2,3,4\}$ and $\mathcal{A}=\{\Phi,\{1\},\{2\},\{3\},\{4\},\{1,3\},\{1,4\},\{2,4\}\}$ where we recall that $\Phi$ denotes the empty set. The CTMC $\{\mathcal{A}(t), t \geq 0\}$ corresponding to this example is given in Figure 4 It can be checked that the transition structure of the CTMC $\{\mathcal{A}(t), t \geq$ $0\}$ satisfies the Kolmogorov Criterion for reversibility (see [24]). Hence, the stationary probability distribution $\pi(\mathcal{A}), \mathcal{A} \in$ $\mathcal{A}$, satisfies the detailed balance equations, $\forall i \in \mathcal{U}_{\mathcal{A}}$,

$$
\pi(\mathcal{A}) \lambda_{i}=\pi(\mathcal{A} \cup\{i\}) \mu_{i},
$$

and the stationary probability distribution has the form

$$
\pi(\mathcal{A})=\left(\prod_{i \in \mathcal{A}} \rho_{i}\right) \pi(\Phi), \quad(\forall \mathcal{A} \in \mathcal{A})
$$

where $\rho_{i}:=\frac{\lambda_{i}}{\mu_{i}}$ and $\pi(\Phi)$ is determined from the normalization equation

$$
\sum_{\mathcal{A} \in \mathcal{A}} \pi(\mathcal{A})=1
$$

Convention: A product $\prod$ over an empty index set is taken to be equal to 1 .

The Second Stage: We now compute the collision probabilities $\gamma_{i}$ 's accounting for inter-cell collisions. Note that $\gamma_{i}$ is conditional on an attempt being made by a node in Cell$i$. Hence, to compute $\gamma_{i}$, we focus only on those states in 


$$
\gamma_{i}=\frac{\sum_{\mathcal{A} \in \mathcal{A}: i \in \mathcal{U}_{\mathcal{A}}} \pi(\mathcal{A})\left[1-\left(1-\beta_{i}\right)^{n_{i}-1} \prod_{j \in \mathcal{N}_{i}: j \in \mathcal{U}_{\mathcal{A}}}\left(1-\beta_{j}\right)^{n_{j}}\right]}{\sum_{\mathcal{A} \in \mathcal{A}: i \in \mathcal{U}_{\mathcal{A}}} \pi(\mathcal{A})} \quad(\forall i \in \mathcal{N})
$$

which Cell- $i$ can attempt. Clearly, Cell- $i$ can attempt in State$\mathcal{A}$ iff it is in back-off in State- $\mathcal{A}$, i.e., iff $i \in \mathcal{U}_{\mathcal{A}}$. In all such states a node in Cell- $i$ can incur intra-cell collisions due the other nodes in Cell- $i$. Furthermore, some (or all) of Cell- $i$ 's neighbors might also be in back-off in State- $\mathcal{A}$. If a neighboring cell, say, Cell- $j, j \in \mathcal{N}_{i}$, is also in back-off in State- $\mathcal{A}$, i.e., if $j \in \mathcal{U}_{\mathcal{A}}$, then a node in Cell- $i$ can incur inter-cell collisions due to the nodes in Cell- $j$. The collision probability $\gamma_{i}$ is then given by Equation 6) (appears at the top of the next page). A formal derivation of Equation 6 is provided in Appendix A

Fixed Point Formulation: Equations 2 26 and $\rho_{i}:=\frac{\lambda_{i}}{\mu_{i}}$ can express the $\gamma_{i}$ 's as functions of only the $\beta_{i}$ 's. Together with Equation 1 they yield an $N$-dimensional fixed point equation where we recall that $N$ is the total number of cells. The $N$ dimensional fixed point equation can be numerically solved to obtain the collision probabilities $\gamma_{i}$ 's, the attempt probabilities $\beta_{i}$ 's and the stationary probabilities $\pi(\mathcal{A})$ 's. In all the cases that we have considered, the fixed point equations yielded unique solutions.

Calculating the Throughputs: The stationary probabilities of the CTMC $\{\mathcal{A}(t), t \geq 0\}$ can provide the fraction of time $x_{i}$ for which Cell- $i$ is unblocked. A cell is said to be unblocked when it belongs to either $\mathcal{A}$ or $\mathcal{U}_{\mathcal{A}}$. Thus, $\forall i \in \mathcal{N}$,

$$
x_{i}=\sum_{\mathcal{A} \in \mathcal{A}: i \in \mathcal{A} \cup \mathcal{U}_{\mathcal{A}}} \pi(\mathcal{A}) .
$$

Definition 5.1: Let $\mathcal{G}_{i}, i \in \mathcal{N}$, denote the subgraph obtained by removing Cell- $i$ and its neighboring cells in $\mathcal{N}_{i}$ from the contention graph $\mathcal{G}$. For a given contention graph $\mathcal{G}$, let $\Delta$ be defined as follows:

$$
\Delta:=\sum_{\mathcal{A} \in \mathcal{A}}\left(\prod_{i \in \mathcal{A}} \rho_{i}\right)
$$

Let $\Delta_{i}$ denote the $\Delta$ corresponding to the subgraph $\mathcal{G}_{i}$.

An important observation which facilitates the computation of the $x_{i}$ 's is given by the following theorem.

Theorem 5.1: The fraction of time $x_{i}$ for which Cell- $i$ is unblocked is given by

$$
x_{i}=\frac{\left(1+\rho_{i}\right) \Delta_{i}}{\Delta}, \quad \forall i \in \mathcal{N} .
$$

Proof: See Appendix B

Let $\Theta_{i}$ denote the aggregate throughput of Cell- $i$ in a given multi-cell network and let $\Theta_{n_{i} \text {,singlecell }}$ denote the aggregate throughput of Cell $i$ if it was an isolated cell containing $n_{i}$ nodes. Both $\Theta_{i}$ and $\Theta_{n_{i}}$,singlecell are in packets/sec. We approximate $\Theta_{i}$ by

$$
\begin{aligned}
\Theta_{i} & =x_{i} \cdot \Theta_{n_{i}, \text { singlecell }} \\
& =\frac{\left(1+\rho_{i}\right) \Delta_{i}}{\Delta} \cdot \Theta_{n_{i}, \text { singlecell }},
\end{aligned}
$$

and $\Theta_{i}$ divided by $n_{i}$ gives the per node throughput $\theta_{i}$ in Cell- $i$, i.e., $\theta_{i}=\frac{\Theta_{i}}{n_{i}}$ (packets/sec).

Discussion 5.2: Equation 10 is justified as follows. If Cell- $i$ is indeed independent of every other cell in the network, it is never blocked and does not incur inter-cell collisions. Then, we have $x_{i}=1$ and $\Theta_{i}=\Theta_{n_{i}}$,singlecell. However, in general, Cell- $i$ gets blocked due to its neighbors for a fraction of time $1-x_{i}$ and remains unblocked for a fraction of time $x_{i}$. The times during which Cell- $i$ is unblocked consists only of the back-off slots and the activities of Cell- $i$ by itself. We approximate the aggregate throughput of Cell- $i$, over the times during which it is unblocked, by $\Theta_{n_{i}}$,singlecell and $\Theta_{n_{i}}$,singlecell multiplied with $x_{i}$ gives the aggregate throughput $\Theta_{i}$ of Cell- $i$ in the multi-cell network.

Clearly, Equation 10 is an approximation since the time wasted in inter-cell collisions have been ignored. However, we prefer to keep the approximation because: (1) it is quite accurate when compared with the simulations (see Section VIl, and (2) it can be efficiently computed since $\Theta_{n_{i} \text {, singlecell }}$ follows from a single cell analysis and the $\Delta$ as well as $\Delta_{i}$ 's can be computed using efficient algorithms [25].

Complexity of the Model: In general, obtaining the state space $\mathcal{A}$ by searching for all possible independent sets $\mathcal{A}$ could be computationally expensive and the complexity grows exponentially with the number of vertices in the contention graph [25]. For realistic topologies, where connectivity in the contention graph is related to distance in the physical network, efficient computation of $\mathcal{A}$ is possible up to several hundred vertices in the contention graph [25]. Thus, a cell level model is extremely helpful in analyzing large-scale WLANs with hundreds of cells since, unlike a node level model, each vertex in the contention graph now represents a cell.

Large $\rho$ Regime: Let $\eta$ (resp. $\eta_{i}$ ) denote the number of Maximum Independent Set: ${ }^{6}$ (MISs) of $\mathcal{G}$ (resp. $\mathcal{G}_{i}$ ) (see Definition 5.1). Then, from Equations 8 and 9 we observe that, as $\rho_{i} \rightarrow \infty, \forall i \in \mathcal{N}$, we have, $x_{i} \rightarrow \frac{\eta_{i}}{\eta}$. The quantity

$$
x_{i}=\frac{\Theta_{i}}{\Theta_{n_{i}, \text { singlecell }}}
$$

can be interpreted as the throughput of Cell- $i$, normalized with respect to Cell- $i$ 's single cell throughput. Thus, as $\rho_{i} \rightarrow \infty$ for all $i \in \mathcal{N}$, the cells that belong to every MIS of the contention graph obtain normalized throughput 1 and the cells that do not

\footnotetext{
${ }^{6} \mathrm{~A}$ maximum independent set of a graph is an independent set of the graph having maximum cardinality.
} 
TABLE I

Single Cell Results: Columns 2-3 Correspond to $n$ Saturated Nodes. COLUMnS 4-7 CORRESPOND TO TCP DOWNLOADS With $n$ STAS

\begin{tabular}{||c|c|c|c|c|c|c||}
\hline$n$ & $\gamma_{a n a}^{\text {sat }}$ & $\begin{array}{c}\theta_{a n a}^{\text {sat }} \\
(\mathrm{pkts} / \mathrm{sec})\end{array}$ & $\gamma_{\text {sim }}^{\text {AP }}$ & $\gamma_{\text {ana }}^{\text {AP }}$ & $\begin{array}{c}\theta_{\text {sim }}^{\text {AP }} \\
(\mathrm{pkts} / \mathrm{sec})\end{array}$ & $\begin{array}{c}\theta_{\text {ana }}^{\text {AP }} \\
(\mathrm{pkts} / \mathrm{sec})\end{array}$ \\
\hline \hline 1 & 0 & 801.78 & 0.0578 & 0.0586 & 454.01 & 456.53 \\
\hline 2 & 0.0586 & 349.94 & 0.0538 & 0.0586 & 456.06 & 456.53 \\
\hline 3 & 0.1077 & 236.09 & 0.0533 & 0.0586 & 456.09 & 456.53 \\
\hline 4 & 0.1473 & 176.63 & 0.0528 & 0.0586 & 456.17 & 456.53 \\
\hline 5 & 0.1812 & 140.29 & 0.0531 & 0.0586 & 456.05 & 456.53 \\
\hline 6 & 0.2100 & 115.89 & 0.0530 & 0.0586 & 456.10 & 456.53 \\
\hline 7 & 0.2348 & 98.43 & 0.0536 & 0.0586 & 456.88 & 456.53 \\
\hline 8 & 0.2565 & 85.35 & 0.0531 & 0.0586 & 456.97 & 456.53 \\
\hline 10 & 0.2927 & 67.11 & 0.0531 & 0.0586 & 456.02 & 456.53 \\
\hline
\end{tabular}

belong to any MIS obtain normalized throughput 0 . Similar observations have also been made in [11]. We further observe that, as $\rho_{i} \rightarrow \infty$ for all $i \in \mathcal{N}$, only an MIS of cells can be active at any point of time since cells that do not belong to any MIS are never active. Since an MIS is always active, as $\rho_{i} \rightarrow \infty$ for all $i \in \mathcal{N}$, the normalized network throughput

$$
\bar{\Theta}:=\sum_{i=1}^{N} x_{i}
$$

is equal to the cardinality $\alpha(\mathcal{G})$ of an MIS of $\mathcal{G} . \alpha(\mathcal{G})$ is also called the independence number of $\mathcal{G}$. Notice that $\alpha(\mathcal{G})$ is a measure of spatial reuse in the network.

\section{B. Extension to TCP Traffic}

We now extend the analysis of Section $\mathrm{V}-\mathrm{A}$ to the more realistic case when users access a local proxy server via persistent TCP connections. Our extension is based on the single cell TCP-WLAN interaction model of [8]. The model proposed in [8] has been shown to be quite accurate when: 1) the local proxy server is connected with the AP by a relatively fast wired LAN such that the AP in the WLAN is the bottleneck, 2) every STA has a single persistent TCP connection, 3) there are no packet losses due to buffer overflow, 4) the TCP timeouts are set large enough to avoid timeout expirations due to Round Trip Time (RTT) fluctuations, and 5) the delayed ACK mechanism is disabled. We keep the above assumptions in this paper.

In [8], the authors propose to model a single cell having an AP and an arbitrary number of STAs with long-lived TCP connections by an "equivalent network" which consists of a saturated AP and a single saturated STA. "This equivalent saturated model greatly simplifies the modeling problem since the TCP flow control mechanisms are now implicitly hidden and the total throughput can be computed using the saturation analysis" [8]. Using the equivalent saturated model of [8], the analysis of Section $\mathrm{V}-\mathrm{A}$ can be applied to the case of persistent TCP connections with $n_{i}=2, \forall i \in \mathcal{N}$.

\section{RESULTS AND DISCUSSION}

We carried out simulations using $n s-2$ [26]. We created the example topologies given in Figure 3(a) 3(d) We chose cell radii and the distances among the cells such that the PBD condition holds. Nodes were randomly placed within the cells. The saturated case was simulated with high rate CBR over UDP connections. For the TCP case, we created one TCP download connection per STA. Each TCP connection was fed by an FTP source with the TCP source agent attached directly to the AP to emulate a local proxy server. The AP buffer and the timeouts were set large enough to avoid buffer losses and timeout expirations. The EIFS deferral and the delayed ACK mechanism were disabled. Each case was simulated 20 times, each run for $200 \mathrm{sec}$ of "simulated time". We report the results for "Basic Access". Similar results were obtained with "RTS/CTS". We took 11 Mbps data rate and packet payloads of 1000 bytes. The function "fsolve()" of MATLAB was used for solving the $N$-dimensional fixed point equation.

Table \ summarizes the results for a single cell. Columns 2-3 (resp. 4-7) correspond to the saturated case (resp. TCP download case) with $n$ saturated nodes (resp. 1 AP and $n$ STAs). The analytical results for the saturated case were obtained using [7] and that for the TCP case were obtained using [8]. These single cell results obtained from known analytical models serve as the basis of our multi-cell results. The analytical throughputs per-node (resp. of AP) in Column 3 (resp. Column 7) multiplied with the $x_{i}$ 's obtained from our multi-cell analysis provide the analytical throughputs per-node (resp. of AP) in the multi-cell cases (see Equation 10).

\section{A. Results for the Saturated Case}

Tables [I-IV summarize the results for the example multicell cases depicted in Figures 3(a) 3(c), respectively, when each cell contains $n$ saturated nodes. Table $\nabla$ summarizes the results for the example case given in Figure 3(d) when Cell- $i$, $1 \leq i \leq 7$, contains $n_{i}=i+1$ saturated nodes. Quantities denoted with a subscript "sim" (resp. "ana") correspond to results obtained from $n s-2$ simulations (resp. fixed point analysis). In each case, $\theta_{\infty}$ represents the throughput per-node obtained by taking $\rho_{i} \rightarrow \infty, \forall i \in \mathcal{N}$. We report only the mean values for our simulation results. The $99 \%$ confidence intervals were observed to be within $5 \%$ of the mean values.

We show the plots corresponding to Table $\mathrm{V}$ in Figures 5 and 6 which compare the collision probability $\gamma$ and the throughput per node $\theta$, respectively. In Figures 5 and 6 we also show the relevant single cell results obtained from Table II i.e., we show the results we would have obtained had the seven cells been mutually independent. Referring Tables $\Pi \mathbf{V}$, and Figures 5 and 6, we make the following observations: O-1.) Collision probabilities (resp. throughputs) in the multicell scenarios are always higher (resp. lower) than the corresponding single cell values (see Figures 5 and 6 because (a) inter-cell collisions can be significant, and (b) due to inter-cell blocking, cells get opportunity to transmit only a fraction of time.

O-2.) Our analytical model is quite accurate (less than $10 \%$ error in most cases) in predicting the collision probabilities and throughputs. However, our model always over-estimates the throughputs since the time wasted in inter-cell collisions have been ignored. Ignoring inter-cell collisions in the first 
TABLE II

Results for the Four Linearly Placed Cells Given in Figure 3(A) WHEN EACH CELl Contains $n=5$ NOdes

\begin{tabular}{||c|c|c|c|c|c||}
\hline $\begin{array}{c}\text { Cell } \\
\text { index }\end{array}$ & $\gamma_{\text {sim }}$ & $\gamma_{\text {ana }}$ & $\begin{array}{c}\theta_{\text {sim }} \\
\text { (pkts/sec) }\end{array}$ & $\begin{array}{c}\theta_{\text {ana }} \\
\text { (pkts/sec) }\end{array}$ & $\begin{array}{c}\theta_{\infty} \\
\text { (pkts/sec) }\end{array}$ \\
\hline \hline 1 & 0.2351 & 0.2399 & 94.48 & 97.41 & 93.53 \\
\hline 2 & 0.3005 & 0.3146 & 41.21 & 46.66 & 46.76 \\
\hline 3 & 0.2999 & 0.3146 & 41.66 & 46.66 & 46.76 \\
\hline 4 & 0.2359 & 0.2399 & 93.99 & 97.41 & 93.53 \\
\hline
\end{tabular}

TABLE III

Results for the Five Linearly Placed Cells Given in Figure 3(B) When EACH Cell Contains $n=5$ Nodes

\begin{tabular}{||c|c|c|c|c|c||}
\hline $\begin{array}{c}\text { Cell } \\
\text { index }\end{array}$ & $\gamma_{\text {sim }}$ & $\gamma_{\text {ana }}$ & $\begin{array}{c}\theta_{\text {sim }} \\
(\mathrm{pkts} / \mathrm{sec})\end{array}$ & $\begin{array}{c}\theta_{\text {ana }} \\
(\mathrm{pkts} / \mathrm{sec})\end{array}$ & $\begin{array}{c}\theta_{\infty} \\
(\mathrm{pkts} / \mathrm{sec})\end{array}$ \\
\hline \hline 1 & 0.1882 & 0.1897 & 129.35 & 131.35 & 140.29 \\
\hline 2 & 0.3321 & 0.3975 & 8.69 & 8.64 & 0 \\
\hline 3 & 0.1892 & 0.1925 & 123.35 & 126.41 & 140.29 \\
\hline 4 & 0.3321 & 0.3975 & 8.72 & 8.64 & 0 \\
\hline 5 & 0.1884 & 0.1897 & 129.31 & 131.35 & 140.29 \\
\hline
\end{tabular}

TABLE IV

Results for the Seven HeXagonally Placed Cells Given in Figure 3(C) when Each Cell Contains $n=10$ Nodes

\begin{tabular}{||c|c|c|c|c|c||}
\hline $\begin{array}{c}\text { Cell } \\
\text { index }\end{array}$ & $\gamma_{\text {sim }}$ & $\gamma_{a n a}$ & $\begin{array}{c}\theta_{\text {sim }} \\
\text { (pkts/sec) }\end{array}$ & $\begin{array}{c}\theta_{a n a} \\
\text { (pkts/sec) }\end{array}$ & $\begin{array}{c}\theta_{\infty} \\
(\mathrm{pkts} / \mathrm{sec})\end{array}$ \\
\hline \hline 1 & 0.2335 & 0.8896 & 0.003 & 0.02 & 0 \\
\hline 2 & 0.3045 & 0.3158 & 31.97 & 32.35 & 33.56 \\
\hline 3 & 0.3061 & 0.3158 & 31.93 & 32.35 & 33.56 \\
\hline 4 & 0.3055 & 0.3158 & 32.05 & 32.35 & 33.56 \\
\hline 5 & 0.3054 & 0.3158 & 31.86 & 32.35 & 33.56 \\
\hline 6 & 0.3070 & 0.3158 & 32.00 & 32.35 & 33.56 \\
\hline 7 & 0.3058 & 0.3158 & 31.95 & 32.35 & 33.56 \\
\hline
\end{tabular}

TABLE V

Results for the Seven ARbitrarily Placed Cells Given in Figure 3(D)] When CELl- $i, 1 \leq i \leq 7$ CONTAINS $n_{i}=i+1$ Nodes

\begin{tabular}{||c|c|c|c|c|c|c||}
\hline $\begin{array}{c}\text { Cell } \\
\text { index } i\end{array}$ & $n_{i}$ & $\gamma_{\text {sim }}$ & $\gamma_{\text {ana }}$ & $\begin{array}{c}\theta_{\text {sim }} \\
(\mathrm{pkts} / \mathrm{sec})\end{array}$ & $\begin{array}{c}\theta_{\text {ana }} \\
(\mathrm{pkts} / \mathrm{sec})\end{array}$ & $\begin{array}{c}\theta_{\infty} \\
(\mathrm{pkts} / \mathrm{sec})\end{array}$ \\
\hline \hline 1 & 2 & 0.0669 & 0.0666 & 320.66 & 325.26 & 349.94 \\
\hline 2 & 3 & 0.1163 & 0.1163 & 216.19 & 219.65 & 236.09 \\
\hline 3 & 4 & 0.2764 & 0.3280 & 12.48 & 12.97 & 0 \\
\hline 4 & 5 & 0.3105 & 0.3318 & 34.29 & 40.20 & 46.76 \\
\hline 5 & 6 & 0.2505 & 0.2585 & 83.77 & 84.92 & 77.26 \\
\hline 6 & 7 & 0.3574 & 0.3787 & 28.67 & 32.40 & 32.81 \\
\hline 7 & 8 & 0.3062 & 0.3139 & 56.87 & 59.21 & 56.90 \\
\hline
\end{tabular}

stage of the model also over-estimates the fraction of time spent in back-off. Thus, the collision probabilities are also over-estimated.

O-3.) The relative mismatch between the analytical model and the simulation is observed to be the worst for cells that remain blocked most of the time. For example, consider the first row of Table IV which corresponds to Cell-1 in Figure 3(c) Since Cell-1 is dependent with respect to every other cell, it obtains very few attempt opportunities in the simulations. Thus, the corresponding simulation results have been averaged over very few samples and are not reliable.

O-4.) Our analytical model correctly identifies the severely blocked cells and works well with either equal or unequal number of nodes per cell.

O-5.) Throughput distribution among the cells can be very unfair even over long periods of time. Furthermore, introduction of a new co-channel cell can drastically alter the throughput

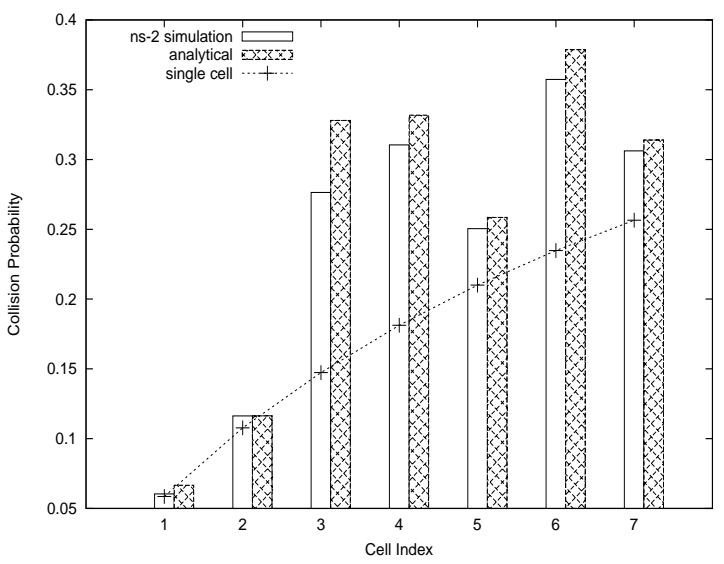

Fig. 5. Comparing collision probability $\gamma$ for the example scenario in Figure 3(d) when Cell- $i, 1 \leq i \leq 7$, contains $n_{i}=i+1$ saturated nodes.

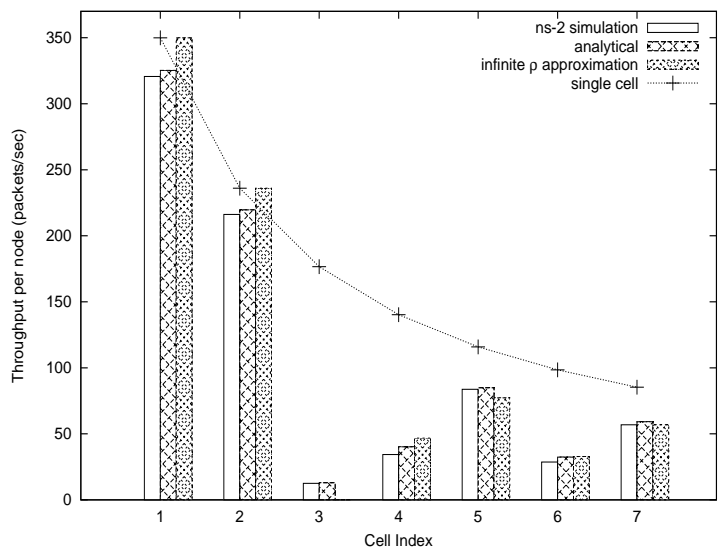

Fig. 6. Comparing throughput per node $\theta$ for the example scenario in Figure 3(d) when Cell- $i, 1 \leq i \leq 7$, contains $n_{i}=i+1$ saturated nodes.

distributions. For example, compare Tables [II and III Cell-2 and Cell- 4 severely get blocked if Cell-5 is introduced to the four cell network given in Figure 3(a)

O-6.) The throughput of a cell cannot be accurately determined based only on the number of interfering cells. Consider, for example, Figure 5 . Cell-3 and Cell-4 each have two neighbors but their per node throughputs $\theta$ are quite different. In particular, $\theta_{4}>\theta_{3}$ even though $n_{3}=4<n_{4}=5$. This is due to Cell-7 which blocks Cell-6 for certain fraction of time during which Cell-4 gets opportunity to transmit whereas Cell-1 and Cell-2 are almost never blocked and Cell-3 is almost always blocked due to Cell-1 and Cell-2. Thus, topology plays the key role and heuristic methods based only on the number of neighbors would fail.

\section{B. Results for the TCP Download Case}

Referring Columns 4-7 of Table \it can be seen that The AP statistics does not change with the number of STAs. Also, O-7.) The collision probability of the AP in a single cell with any number of STAs is approximately equal to the collision 
TABLE VI

RESULTS FOR THE AP CORRESPONDING TO FIGURE 3(A) WHEN EACH CEll CONTAINS 1 AP AND $n=5$ STAS

\begin{tabular}{||c|c|c|c|c|c||}
\hline $\begin{array}{c}\text { Cell } \\
\text { index }\end{array}$ & $\gamma_{\text {sim }, A P}$ & $\gamma_{\text {ana }, A P}$ & $\begin{array}{c}\theta_{\text {sim, }}, A P \\
\text { (pkts/sec) }\end{array}$ & $\begin{array}{c}\theta_{\text {ana }, A P} \\
(\mathrm{pkts} / \mathrm{sec})\end{array}$ & $\begin{array}{c}\theta_{\infty} \\
(\mathrm{pkts} / \mathrm{sec})\end{array}$ \\
\hline \hline 1 & 0.1038 & 0.1033 & 306.33 & 318.73 & 304.35 \\
\hline 2 & 0.1560 & 0.1574 & 153.16 & 169.18 & 152.18 \\
\hline 3 & 0.1555 & 0.1574 & 153.06 & 169.18 & 152.18 \\
\hline 4 & 0.1038 & 0.1033 & 306.41 & 318.73 & 304.35 \\
\hline
\end{tabular}

TABLE VII

RESULTS FOR THE AP CORRESPONDING TO FIGURE 3(B) WHEN EACH Cell Contains 1 AP AND $n=5$ STAS

\begin{tabular}{||c|c|c|c|c|c||}
\hline $\begin{array}{c}\text { Cell } \\
\text { index }\end{array}$ & $\gamma_{\text {sim }, A P}$ & $\gamma_{\text {ana }, A P}$ & $\begin{array}{c}\theta_{\text {sim }, A P} \\
(\mathrm{pkts} / \mathrm{sec})\end{array}$ & $\begin{array}{c}\theta_{\text {ana }, A P} \\
(\mathrm{pkts} / \mathrm{sec})\end{array}$ & $\begin{array}{c}\theta_{\infty} \\
(\mathrm{pkts} / \mathrm{sec})\end{array}$ \\
\hline \hline 1 & 0.0728 & 0.0775 & 381.21 & 387.16 & 456.53 \\
\hline 2 & 0.1793 & 0.1950 & 75.16 & 85.62 & 0 \\
\hline 3 & 0.0744 & 0.0832 & 340.24 & 346.47 & 456.53 \\
\hline 4 & 0.1786 & 0.1950 & 75.23 & 85.62 & 0 \\
\hline 5 & 0.0728 & 0.0775 & 381.15 & 387.16 & 456.53 \\
\hline
\end{tabular}

TABLE VIII

RESUltS FOR THE AP CORRESPONDING TO FIGURE 3(D) WHEN EACH Cell Contains 1 AP AND $n=5$ STAS

\begin{tabular}{||c|c|c|c|c|c||}
\hline $\begin{array}{c}\text { Cell } \\
\text { index }\end{array}$ & $\gamma_{\text {sim, AP }}$ & $\gamma_{\text {ana, }, P}$ & $\begin{array}{c}\theta_{\text {sim }, A P} \\
(\mathrm{pkts} / \mathrm{sec})\end{array}$ & $\begin{array}{c}\theta_{\text {ana, } A P} \\
(\mathrm{pkts} / \mathrm{sec})\end{array}$ & $\begin{array}{c}\theta_{\infty} \\
(\mathrm{pkts} / \mathrm{sec})\end{array}$ \\
\hline \hline 1 & 0.0610 & 0.0670 & 421.70 & 425.83 & 456.53 \\
\hline 2 & 0.0604 & 0.0670 & 421.92 & 425.83 & 456.53 \\
\hline 3 & 0.2010 & 0.2528 & 33.79 & 38.50 & 0 \\
\hline 4 & 0.1561 & 0.1685 & 141.80 & 156.41 & 152.18 \\
\hline 5 & 0.0987 & 0.1028 & 317.39 & 329.06 & 304.35 \\
\hline 6 & 0.1551 & 0.1644 & 158.55 & 172.64 & 152.18 \\
\hline 7 & 0.1061 & 0.1099 & 301.15 & 314.10 & 304.35 \\
\hline
\end{tabular}

probability in a single cell with two saturated nodes. This can be verified by comparing Columns 4 and 5 with Row 2 Column 2 in Table 【

O-8.) The AP throughput does not change with the number of STAs. In fact, the AP throughput with any number of STAs is equal to the per node throughput in a single cell containing two saturated nodes with payload size $\frac{L_{T C P-D A T A}+L_{T C P-A C K}}{2}$ where $L_{T C P-D A T A}$ and $L_{T C P}-A C K$ denote the size of TCP DATA and TCP ACK packets.

Observations O-7 and O-8 are well-known and they form the basis for the equivalent saturated model of [8]. Observation O-7 has led to the conclusion in [3] that the collision probability of the nodes in a WLAN containing $m$ mutually interfering (i.e., dependent) APs is equal to the collision probability in a single cell containing $2 m$ saturated nodes. Tables VI-VIII summarize the AP statistics for the topologies in Figures 3(a). 3(b) and 3(d), respectively. Since the AP statistics does not change with the number of STAs, we report the results with $n=5$ STAs in each case. We show the plots corresponding to Table VIII in Figures 7 and 8 which compare the collision probability $\gamma$ and the throughput per node $\theta$, respectively. Referring Tables VI VIII, and Figures 7 and 8, we conclude that the foregoing observations for the saturated case carry over to TCP-controlled long file transfers as well. Furthermore, we generalize the conclusion of [3] as follows:

O-9.) The collision probability in a WLAN depends, not only

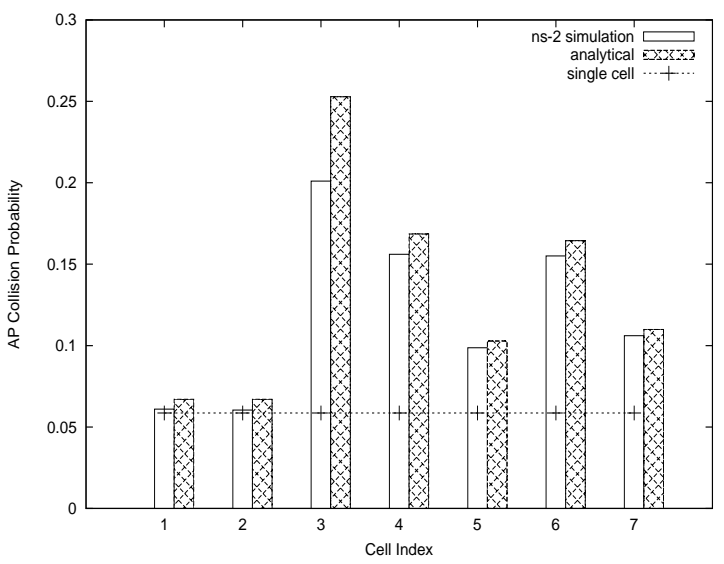

Fig. 7. Comparing collision probability $\gamma$ for the example scenario in Figure 3(d) when each cell contains an AP and $n=5$ STAs. STAs are downloading long files through their respective APs using TCP connections.

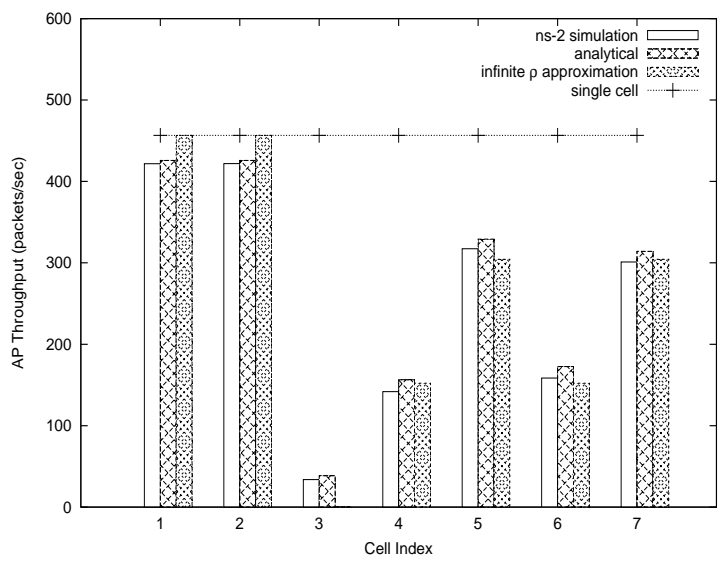

Fig. 8. Comparing throughput per node $\theta$ for the example scenario in Figure 3(d) when each cell contains an AP and $n=5$ STAs. STAs are downloading long files through their respective APs using TCP connections.

on the number of interfering APs but also on the fraction of time when the neighboring APs can cause collisions. In general, collision probability does not grow as twice the number of interfering APs.

Referring Tables VIVIII we extend the validity of the equivalent saturated model of [8] as follows:

O-10.) The equivalent saturated model of [8] proposed in the context of a single cell, preserves its desirable properties, i.e., it predicts the AP statistics quite well when extended to a multi-cell WLAN that satisfies the PBD condition.

\section{Variation with $\rho$}

Till now, we have discussed the results corresponding to the payload size of 1000 bytes. We now examine how the results vary with the payload size. Figures 9 and 10 depict the variation with payload size of analytically computed collision probabilities and normalized cell throughputs, respectively, for the seven cell network of Figure 3(d) when each cell contains 


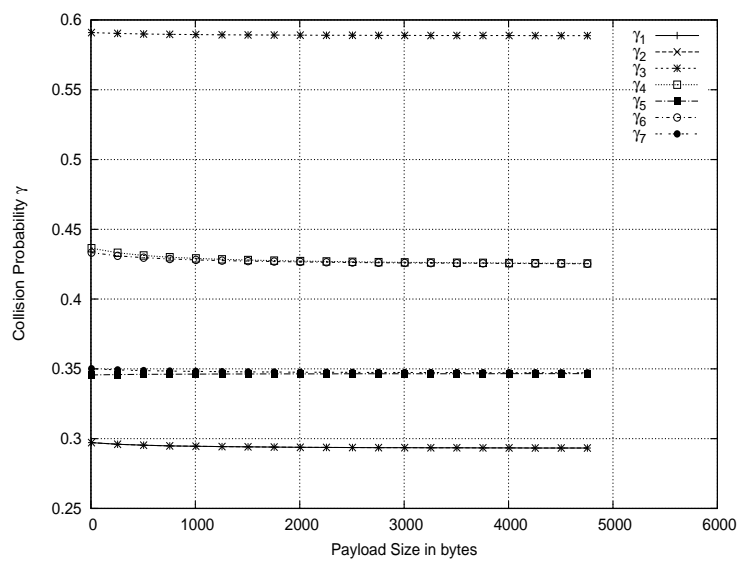

Fig. 9. Variation of collision probabilities with payload size for the seven cell network in Figure 3(d) when each cell contains $n=10$ saturated nodes.

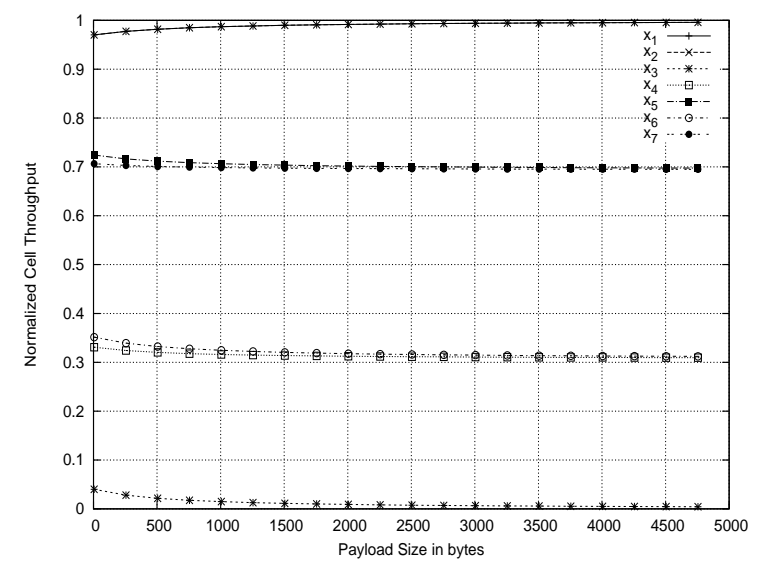

Fig. 10. Variation of normalized cell throughputs with payload size for the seven cell network in Figure 3(d) when each cell contains $n=10$ saturated nodes.

$n=10$ saturated nodes. From Figures 9 and 10, we observe that:

O-11.) The results are largely insensitive to the variation in payload size. Moreover, as the payload size increases, the normalized cell throughputs become closer to the normalized throughputs under the infinite $\rho$ approximation, i.e., they become closer to $x_{1}=x_{2}=1, x_{3}=0, x_{4}=x_{6}=$ $\frac{1}{3}, x_{5}=x_{7}=\frac{2}{3}$. Hence, except for very small payload sizes, the infinite $\rho$ approximation can be expected to provide fairly accurate predictions. Furthermore, for sufficiently large payload sizes, we have $\bar{\Theta} \approx \alpha(\mathcal{G})$.

\section{A Simple Design EXAmple}

We consider a 12-cell network as shown in Figure 11 and apply our throughput model to compare three different channel assignments as shown in Figures 12(a) 12(c) Notice that the "logical" contention graphs for the three assignments in Figures 12(a) 12(c) are different from the "physical" contention graph of the network shown in Figure 11 which is based on the physical separation among the cells. Two dependent cells

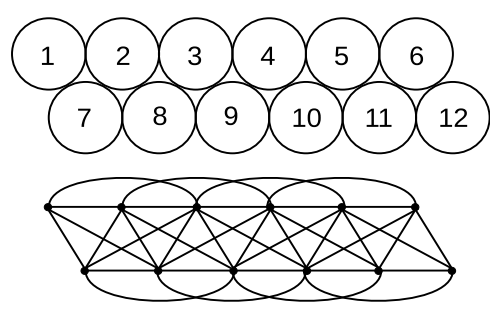

Fig. 11. A 12-cell network: Cell indices have been marked. Also shown is the cell level contention graph assumed for the network.

become independent if they are assigned different channels and the corresponding edge will be missing in the logical contention graph. Our objective is to determine the best, among the three assignments, in terms of the normalized network throughput $\bar{\Theta}$ (see Equation 11) and the fairness index reported in [27]. Considering fairness at the cell level, the fairness index $J$ is given by [27]

$$
J:=\frac{\left(\sum_{i=1}^{N} x_{i}\right)^{2}}{N\left(\sum_{i=1}^{N} x_{i}^{2}\right)},
$$

where we recall that $x_{i}$ is the normalized throughput of Cell$i$. Note that, $0 \leq J \leq 1$, and higher values of $J$ imply more fairness.

Observe that co-channel cells in the first assignment have topology as in Figure 3(a). There are three subsystem of co-channel cells each consisting of four cells. The infinite $\rho$ approximation predicts that, the two cells at the middle (resp. at the end) for each subsystem of co-channel cells would obtain normalized throughput $\approx \frac{1}{3}$ (resp. $\approx \frac{2}{3}$ ). The normalized network throughput for the first assignment is 6 and the fairness index is 0.45 . In the second assignment, each cell obtains normalized throughput $\approx \frac{1}{3}$. The normalized network throughput for the second assignment is 4 and the fairness index is 1 . Thus, the fairness has attained its maximum in the second assignment. However, this assignment is not desirable since $\bar{\Theta}$ for this assignment is only $\frac{2}{3}$ of $\bar{\Theta}$ in the first assignment. In the third assignment, each cell obtains normalized throughput $\approx \frac{1}{2}$. The normalized network throughput for the third assignment is 6 and the fairness index is equal to 1. Clearly, the third assignment is the best one among the three.

The above design example indicates that for relatively smaller networks, we could apply our analytical model to examine all possible assignments. The infinite $\rho$ approximation could provide fairly accurate comparisons among the assignments and the fixed point analysis could be applied to obtain more accurate comparisons. However, the fixed point analysis would require more computation. Furthermore, any objective other than the ones taken above could be considered. 


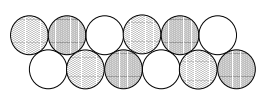

$\therefore \times$.

(a)

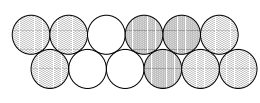

$\nabla \wedge \vee \wedge$

(b)

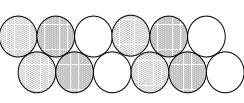

$\cdot \cdot \cdot \cdot \cdot$

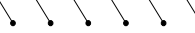

(c)

Fig. 12. Comparing three channel assignments for a 12-cell network: The "logical" cell level contention graph are also shown for each assignment.

\section{Applying the Analytical Model along With a LEARNING ALGORITHM}

In the 12-cell network of Figure 11, with 3 non-overlapping channels, we need to examine $3^{12}$ possibilities to determine the optimal assignment. It is also not clear if the normalized network throughput for the 12-cell network can be actually more than 6 with some other assignment. Under the infinite $\rho$ approximation, the maximum value of $\bar{\Theta}$ is equal to the maximum independence number over all possible logical contention graphs. Clearly, the problem of finding an assignment that maximizes $\bar{\Theta}$ is NP-hard and, in general, it is not desirable to examine all the possibilities.

We now demonstrate how our analytical model could be applied along with a Learning Automata (LA) algorithm called the Linear Reward-Inaction $\left(L_{R-I}\right)$ algorithm [28] to obtain optimal channel assignments. For every cell $i \in \mathcal{N}$, we maintain an $M$-dimensional probability vector $\boldsymbol{p}_{i}$ where $M$ denotes the number of available channels. The learning algorithm proceeds in steps. Let $k=0,1,2, \cdots$ be the step indices. At each step $k$, a channel is selected for Cell- $i, i \in \mathcal{N}$, according to the probability distribution

$$
\boldsymbol{p}_{i}(k):=\left(p_{i, 1}(k), p_{i, 2}(k), \cdots, p_{i, M}(k)\right),
$$

where

$$
\begin{aligned}
p_{i, j}(k):= & \text { the probability that Channel- } j \text { is selected for } \\
& \text { Cell- } i \text { at step } k .
\end{aligned}
$$

Let $\boldsymbol{c}(k)=\left(c_{1}(k), c_{2}(k), \cdots, c_{N}(k)\right)$ be the channel assignment at step $k$, where

$$
c_{i}(k):=\text { the channel selected for Cell- } i \text { at step } k .
$$

Given a channel assignment $\boldsymbol{c}$, we can obtain the logical contention graph $\mathcal{G}(\boldsymbol{c})$ and applying our throughput model, we can compute the normalized throughput $x_{i}(\boldsymbol{c})$ of every cell $i$ with the assignment $\boldsymbol{c}$. We define the sum utility for an assignment $c$ by

$$
U(\boldsymbol{c}):=\sum_{i=1}^{N} u\left(x_{i}(\boldsymbol{c})\right),
$$

where $u(\cdot)$ is some suitably defined increasing concave function. For a given $u(\cdot)$, we compute the sum utility at step $k$ by

$$
U(\boldsymbol{c}(k))=\sum_{i=1}^{N} u\left(x_{i}(\boldsymbol{c}(k))\right),
$$

and apply the $L_{R-I}$ algorithm which consists of the following steps [28]:

1. Begin with $p_{i, j} \in(0,1), \forall i \in \mathcal{N}, j=1,2, \cdots, M$, such that $\boldsymbol{p}_{i} \cdot \mathbf{1}=1$, where $\mathbf{1}$ is the $M$-dimensional vector with all components equal to 1 .

2. Update the probability vectors of cell $i, \forall i \in \mathcal{N}$, as

$$
\boldsymbol{p}_{i}(k+1)=\boldsymbol{p}_{i}(k)+b U(\boldsymbol{c}(k))\left(\boldsymbol{\delta}_{c_{i}(k)}-\boldsymbol{p}_{i}(k)\right),
$$

where $\boldsymbol{\delta}_{j}$ denotes the $M$-dimensional probability vector with unit mass on Channel- $j$ and $0<b<1$.

The parameter $b$ is called the learning parameter or the stepsize parameter which determines the convergence properties of the algorithm; with smaller $b$ convergence is slower but the algorithm may not converge to the desired optimum if $b$ is not small enough [28]. Notice that, to ensure nonnegativity of the probability vectors after every update with any $b \in(0,1)$, the sum utility $U$ must satisfy $0 \leq U \leq 1$. To maximize $\bar{\Theta}=\sum_{i=1}^{N} x_{i}$, we take the average normalized network throughput

$$
U_{\bar{\Theta}}:=\frac{1}{N} \sum_{i=1}^{N} x_{i}
$$

as the sum utility $U$. Note that, $0 \leq U_{\bar{\Theta}} \leq 1$. Other utility functions can also be considered provided that $0 \leq U \leq 1$. Define

$$
\mathbf{P}:=\left(\boldsymbol{p}_{1}, \boldsymbol{p}_{2}, \cdots, \boldsymbol{p}_{N}\right) .
$$

Using the terminology of [28], the super vector $\mathbf{P}$ will be called a strategy for the channel assignment problem. A strategy $\mathbf{P}$ such that, $\forall i \in \mathcal{N}, p_{i, j}=1$ for some $j$, $j=1,2, \cdots, M$, will be called a pure strategy. A strategy $\mathbf{P}$ that is not a pure strategy is called a mixed strategy. Under the $L_{R-I}$ algorithm, $\{\mathbf{P}(k), k \geq 0\}$ is a Markov process with the pure strategies as the only absorbing states. Thus, in practice, the $L_{R-I}$ algorithm always converges to a pure strategy rather than to a mixed strategy [28]. Furthermore, by Theorem 4.1 of [28], the $L_{R-I}$ algorithm always converges to one of the Nash equilibria. Thus, in practice, channel assignment by the $L_{R-I}$ algorithm always provides an assignment $c^{*}$ which is one of the Nash equilibria in pure strategies in the sense that

$$
U\left(\boldsymbol{c}^{*}\right) \geq U(\boldsymbol{c}),
$$

for all $c$ that differs from $c^{*}$ by exactly one element, i.e., changing the channel of one of the cells in the assignment $c^{*}$ does not increase the utility $U$. 


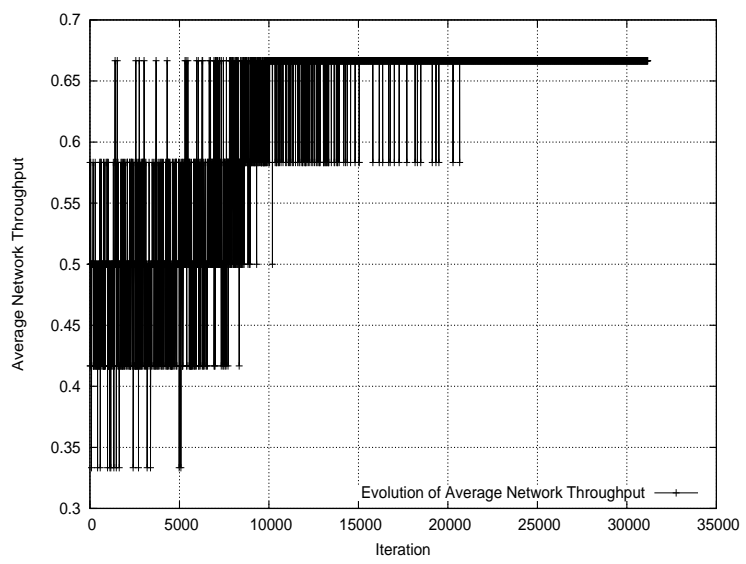

Fig. 13. Evolution of the average normalized network throughput $U_{\bar{\Theta}}$ under the $L_{R-I}$ algorithm with $M=3$ channels and $b=0.01$ for the 12-cell network in Figure 11

\section{A. Results for Channel Assignment by the $L_{R-I}$ Algorithm}

Figure 13 shows the evolution of the average normalized network throughput $U_{\bar{\Theta}}$ under the $L_{R-I}$ algorithm with $M=3$ channels and $b=0.01$ for the 12 -cell network in Figure 11. We bagan with uniform unbiased probability vectors $\boldsymbol{p}_{i}=\left(\frac{1}{3}, \frac{1}{3}, \frac{1}{3}\right), \forall i \in \mathcal{N}$. The algorithm converged to the assignment $c_{1}=c_{6}=c_{9}=1, c_{4}=c_{7}=c_{12}=$ $3, c_{2}=c_{3}=c_{5}=c_{8}=c_{10}=c_{11}=2$. Notice that $U_{\bar{\Theta}}$ coverges to $\frac{2}{3}$ which corresponds to $\bar{\Theta}=8$. It can be verified that this is the maximum possible value that $\bar{\Theta}$ can take for the given network with 3 channels. We found that the $L_{R-I}$ algorithm always converges to a globally optimum solution if started with uniform unbiased probability vectors and if $b$ is sufficiently small. However, starting with probability vectors that are highly biased towards some assignment or if $b$ is not small enough we do not obtain a globally optimum solution. We demonstrate this through Figures 14]17 for the 7-cell example in Figure 3(d) with $M=2$ channels.

Begining with unbiased probability vectors and $b=0.01$ the algorithm may converge to a global optimum with $U(\bar{\Theta})=$ 1 or $\bar{\Theta}=7$ (Figure 14). In Figure 15, we show that, with $b=0.01$, the algorithm may also converge to a solution that is not a global optimum. In fact, for the case in Figure 15, the algorithm converges to the Nash equilibrium $c_{1}=c_{2}=$ $c_{5}=c_{6}=1, c_{3}=c_{4}=c_{7}=2$. With $b=0.001$, and begining with unbiased uniform probability vectors, we observed that the algorithm always converges to a global optimum. With $b=0.001$, the algorithm converges to a global optimum even when we begin with probability vectors biased towards the assignment $c_{1}=c_{2}=c_{5}=c_{6}=1, c_{3}=c_{4}=c_{7}=2$ (Figure 16). However, when the initial probability vectors are highly biased towards the assignment $c_{1}=c_{2}=c_{5}=c_{6}=1, c_{3}=$ $c_{4}=c_{7}=2$, the algorithm converges to the biased assignment which is not a global optimum (Figure 17).

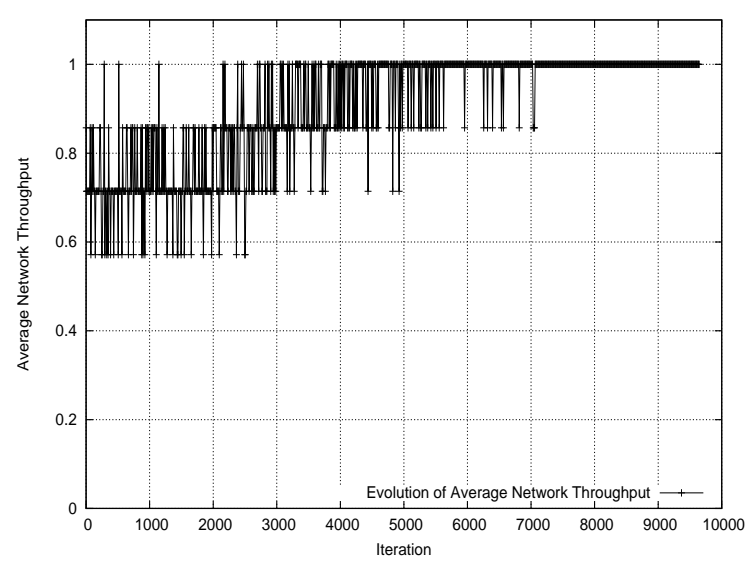

Fig. 14. Evolution of the average normalized network throughput $U_{\bar{\Theta}}$ with $M=2$ channels, $b=0.01$, and $p_{i 1}=p_{i 2}=0.5, i=$ $1,2, \cdots, 7$ for the 7 -cell example in Figure $3(\mathrm{~d})$ In this example, the $L_{R-I}$ algorithm converges to a global optimum.

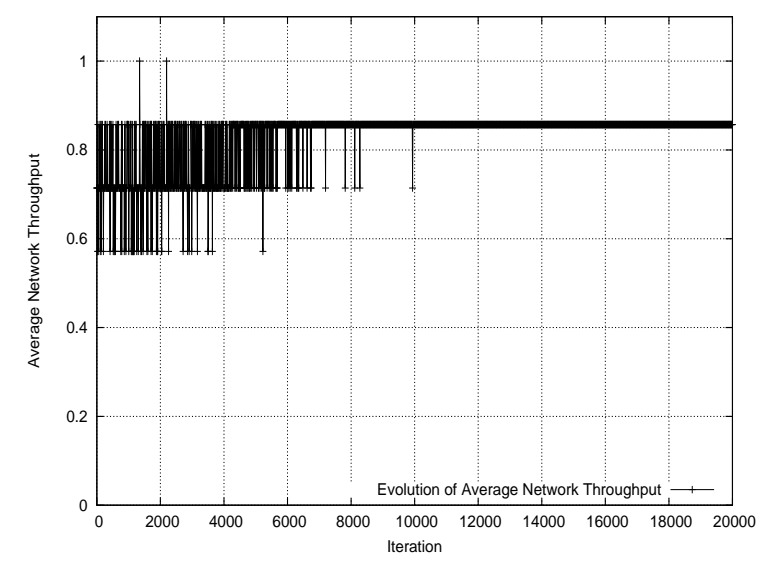

Fig. 15. Evolution of the average normalized network throughput $U_{\bar{\Theta}}$ with $M=2$ channels, $b=0.01$, and $p_{i 1}=p_{i 2}=0.5, i=$ $1,2, \cdots, 7$ for the 7 -cell example in Figure 3(d) In this example, the $L_{R-I}$ algorithm converges to a local optimum.

\section{A Simple And Fast Algorithm for Maximizing Aggregate Throughrut}

We observe that the $L_{R-I}$ algorithm takes a large number of iterations to converge and guarantees covergence only to Nash equilibria. The Linear Reward-Penalty $\left(L_{R-P}\right)$ algorithm of [22] and the simulated annealing algorithm of [23] guarantee convergence to a globally optimum solution as the number of iterations goes to infinity. A greedy version of simulated annealing algorithm in [23] is relatively faster but still takes a large number of iterations to converge and guarantees convergence only to locally optimum solutions. To maximize the normalized network throughput $\bar{\Theta}$ we now propose a simple and fast decentralized algorithm which can be easily implemented in real networks. We form a contention graph $\mathcal{G}$ in which every completely dependent pair of cells are neighbors (since we have not yet assigned channels) and our objective is to transform $\mathcal{G}$ to $\mathcal{G}(\boldsymbol{c})$ by an assignment $\boldsymbol{c}$ so 


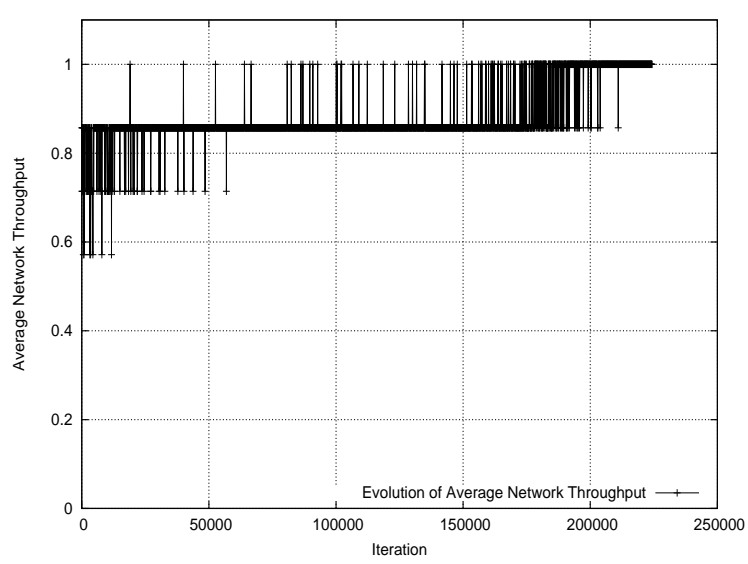

Fig. 16. Evolution of the average normalized network throughput $U_{\bar{\Theta}}$ with $M=2$ channels, $b=0.001$, and $p_{11}=p_{21}=p_{32}=$ $p_{42}=p_{51}=p_{61}=p_{72}=0.8$ for the 7-cell example in Figure 3(d) In this example, the $L_{R-I}$ algorithm converges to a global optimum.

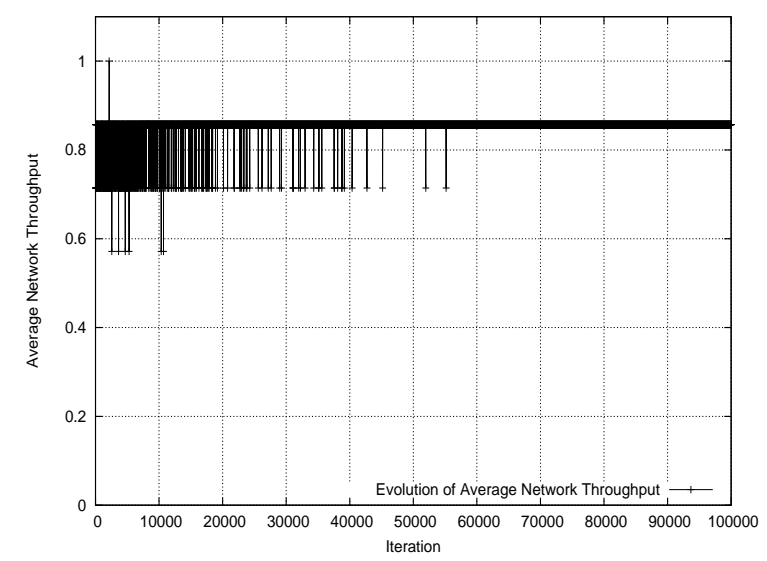

Fig. 17. Evolution of the average normalized network throughput $U_{\bar{\Theta}}$ with $M=2$ channels, $b=0.001$, and $p_{11}=p_{21}=p_{32}=$ $p_{42}=p_{51}=p_{61}=p_{72}=0.9$ for the 7-cell example in Figure 3(d) In this example, the $L_{R-I}$ algorithm converges to a local optimum.

that $\alpha(\mathcal{G}(\boldsymbol{c}))$ is maximized. As noted in Observation 0.11, for sufficiently large packet sizes, we have $\bar{\Theta} \approx \alpha(\mathcal{G})$. Hence, maximizing $\alpha(\mathcal{G}(\boldsymbol{c}))$ would maximize $\bar{\Theta}$.

Maximal Independent Set Algorithm (MISA) : We propose the following channel assignment algorithm:

(1) Choose a maximal independent set of cells, assign them Channel-1 and remove them from the graph.

(2) Increment the channel index and repeat the procedure on the residual graph until one channel is left.

(3) Assign Channel- $M$ to all the cells in the residual graph after $M-1$ steps.

Notice that MISA takes only $M$ steps where $M$ denotes the number of available channels.

Theorem 9.1: The channel assignments by MISA are Nash equilibria in pure strategies for the objective of maximizing normalized network throughput as $\rho_{i} \rightarrow \infty, \forall i \in \mathcal{N}$.

Proof: Suppose that $N_{j}$ cells are assigned Channel- $j$ in Step- $j, 1 \leq j \leq M-1$. Let $\mathcal{G}_{M-1}$ be the residual graph after $M-1$ steps. Then, we have $\bar{\Theta}=\sum_{j=1}^{M-1} N_{j}+\alpha\left(\mathcal{G}_{M-1}\right)$ since, for any $j, 1 \leq j \leq M-1$, cells that have been assigned Channel- $j$, are independent. Suppose now that the channel of a cell on Channel- $j, j \neq M$, is changed to Channel- $k$, $k \neq j$. Then the aggregate normalized throughput of the cells on Channel- $j$ decreases by 1 but the aggregate normalized throughput of the cells on Channel- $k$ can increase by at most 1. Hence, $\bar{\Theta}$ cannot increase. Suppose that the channel of a cell on Channel- $M$ is changed to Channel- $l, 1 \leq l \leq M-1$. Clearly, the cell on Channel- $M$ is dependent w.r.t. at least one of the cells on Channel- $l$ since the $N_{l}$ cells that are already on Channel-l, $1 \leq l \leq M-1$, form a maximal independent set. Hence, the aggregate normalized throughput of the cells on Channel-l does not change but the aggregate normalized throughput of the cells on Channel- $M$ can only decrease. Hence, $\bar{\Theta}$ cannot increase by changing the channel of one of the cells.

Implementation: MISA can be implemented in a decentralized manner as follows. APs sample random back-offs using a contention window $W$ and contend for accessing the medium using Channel-1. When an AP wins the contention it keeps transmitting broadcast packets separated by Short Inter Frame Space (SIFS) for some duration $T>>\sigma W$ where we recall that $\sigma$ is the duration of a back-off slot. This emulates the infinite $\rho$ situation since and AP after wining the contention does not relinquishes the control over its local medium. We had observed that, as $\rho_{i} \rightarrow \infty, \forall i \in \mathcal{N}$, only the cells that belong to an MIS obtain non-zero normalized throughputs. But this holds only in an ensemble average sense. If an AP, after wining the contention, does not relinquishes the control over its local medium, in a particular sample path, a maximal independent set of APs (which may not be an MIS) would grab the channel during $T$. This is not surprising since with infinite $\rho_{i}$ 's, the CTMC $\{\mathcal{A}(t), t \geq 0\}$ becomes absorbing with the maximal independent sets of cells as the only absorbing states and we cannot expect the time average to be equl to the ensemble average.

At time $T$, APs that could transmit consecutive broadcast packets stop contending until time $(M-1) \times T$ and APs that remain blocked switch to Channel-2, sample fresh backoffs and keep contending until $2 T$ and so on. APs that remain blocked throughout the duration $(M-1) \times T$ stick to Channel- $M$. Thus, in every time duration $T$, a maximal independent set of APs would be assigned a channel. Normal network operation can begin after time $(M-1) \times T$. In addition, if there is a central controller to which the APs can communicate, MISA can be repeated several times and the central controller, which obtains the global view of the channel assignments, can choose the best among the solutions provided by MISA. In absence of centralized control, MISA can be invoked periodically. MISA can be easily implemented in real networks in a completely decentralized manner if the number of channels for every AP is the same and known. MISA also requires loose synchronization among the APs. 


\section{Conclusions And Future Work}

In this paper, we identified a Pairwise Binary Dependence (PBD) condition that allows a scalable cell level modeling of WLANs. The PBD condition is likely to hold at higher PHY rates and denser AP deployments. We developed a cell level model both under saturation condition and for long-lived TCP transfers. Our analytical model was shown to be quite accurate, insightful and capable of comparing few design alternatives. Thus, we believe that our modeling framework is a significant step toward gaining "first-cut" analytical understanding of WLANs having a dense deployment of APs. We demonstrated how our analytical model could be applied along with the Linear Reward-Inaction learning algorithm for optimal channel assignment. We also proposed a simple decentralized algorithm called MISA which can provide channel assignments that are Nash equilibria in pure strategies in only as many steps as there are channels. In this paper, we considered maximizing the normalized network throughput. Developing simple and practical algorithms for general objective functions is a topic of our ongoing research.

\section{REFERENCES}

[1] "Wireless LAN Medium Access Control (MAC) and (PHY) Layer Specifications, ANSI/IEEE Std 802.11, 1999 Edition."

[2] R. Murty, J. Padhye, R. Chandra, A. Wolman, and B. Zill, "Designing High Performance Enterprise Wi-Fi Networks," in 5th USENIX Symposium on Networked Systems Design and Implementation NSDI'08, 2008.

[3] M. A. Ergin, K. Ramachandran, and M. Gruteser, "An Experimental Study of Inter-cell Interference Effects on System Performance in Unplanned Wireless Deployments," Computer Networks, 2008, accepted manuscript.

[4] P. Belanger, "Enterprise Wireless LAN Scale Testing," Novarum Inc., Tech. Rep., 2007.

[5] G. Bianchi, "Performance Analysis of the IEEE 802.11 Distributed Coordination Function," IEEE Journal on Selected Areas in Communications, vol. 18, no. 3, pp. 535-547, March 2000.

[6] F. Cali, M. Conti, and E. Gregori, "Dynamic Tuning of the IEEE 802.11 Protocol to Achieve a Theoretical Throughput Limit," IEEE/ACM Transactions on Networking, vol. 8, no. 6, pp. 785-799, December 2000.

[7] A. Kumar, E. Altman, D. Miorandi, and M. Goyal, "New insights from a fixed point analysis of single cell IEEE 802.11 WLANs," IEEE/ACM Transactions on Networking, vol. 15, no. 3, pp. 588-601, June 2007, also appeared in INFOCOM 2005.

[8] R. Bruno, M. Conti, and E. Gregori, "An accurate closed-form formula for the throughput of long-lived TCP connections in IEEE 802.11 WLANs," Computer Networks, vol. 52, pp. 199-212, 2008.

[9] R. Boorstyn, A. Kershenbaum, B. Maglaris, and V. Sahin, "Throughput Analysis in Multihop CSMA Packet Radio Networks," IEEE Transactions on Communications, vol. 35, no. 3, pp. 267-274, March 1987.

[10] M. Garetto, T. Salonidis, and E. W. Knightly, "Modeling Per-flow Throughput and Capturing Starvation in CSMA Multi-hop Networks," IEEE/ACM Transactions on Networking, to appear, Also appeared in INFOCOM 2006.

[11] X. Wang and K. Kar, "Throughput Modeling and Fairness Issues in CSMA/CA Based Ad Hoc Networks," in IEEE INFOCOM, 2005.

[12] M. Durvy, O. Dousse, and P. Thiran, "Border Effects, Fairness, and Phase Transitions in Large Wireless Networks," in INFOCOM'08.

[13] S. Roy, H. Ma, and R. Vijayakumar, "Optimizing 802.11 Wireless Mesh Network Performance Using Physical Carrier Sensing," University of Washington, Tech. Rep. UWEETR-2006-0005, 2006.

[14] H. Q. Nguyen, F. Baccelli, and D. Kofman, "A Stochastic Geometry Analysis of Dense IEEE 802.11 Networks," in IEEE INFOCOM'07.

[15] T. Bonald, A. Ibrahim, and J. Roberts, "Traffic Capacity of Multi-Cell WLANs," in ACM SIGMETRICS'08.

[16] X. Yang and N. Vaidya, "On Physical Carrier Sensing in Wireless Ad Hoc Networks," in IEEE INFOCOM 2005.
[17] K. K. Leung and B.-J. J. Kim, "Frequency Assignment for Multi-Cell IEEE 802.11 Wireless Networks," in Proceedings of VTC'2003.

[18] A. Akella, G. Judd, S. Seshan, and P. Steenkiste, "Self-Management in Chaotic Wireless Deployments," in ACM Mobicom'05, 2005.

[19] V. P. Mhatre, K. Papagiannaki, and F. Baccelli, "Interference mitigation through power control in high density 802.11 wlans," in IEEE INFOCOM'07, 2007.

[20] A. Mishra, V. Brik, S. Banerjee, and A. S. W. Arbaugh, "A Clientdriven Approach for Channel Management in Wireless LANs," in IEEE INFOCOM'06, 2006.

[21] A. Mishra, V. Shrivastava, D. Agrawal, S. Banerjee, and S. Ganguly, "Distributed channel management in uncoordinated wireless environments," in ACM Mobicom'06, 2006, pp. 170-181.

[22] D. J. Leith and P. Clifford, "A Self-Managed Distributed Channel Selection Algorithm for WLANs," in Proceedings of RAWNET'06.

[23] B. Kauffmann, F. Baccelli, and A. Chaintreau, "Measurement-Based Self Organization of Interfering 802.11 Wireless Access Networks," in IEEE INFOCOM'07, 2007.

[24] F. P. Kelly, Reversibility in Stochastic Networks. John Wiley, 1979.

[25] A. Kershenbaum, R. R. Boorstyn, and M.-S. Chen, "An Algorithm for Evaluation of Throughput in Multihop Packet Radio Networks with Complex Topologies," IEEE Journal on Selected Areas in Communications, vol. SAC-5, no. 6, pp. 1003-1012, July 1987.

[26] S. McCanne and S. Floyd., "The ns Network Simulator." http://www.isi.edu/nsnam/ns/.

[27] C. E. Koskal, H. Kassab, and H. Balakrishnan, "An Analysis of ShortTerm Fairness in Wireless Media Access Protocols," in ACM SIGMETRICS, 2000.

[28] P. S. Sastry, V. V. Phansalkar, and M. A. L. Thathachar, "Decentralized Learning of Nash Equilibria in Multi-Person Stochastic Games With Incomplete Information," IEEE Transactions on Systems, Man, and Cybernetics, vol. 24, no. 5, pp. 769-777, 1994. 


\section{APPENDIX A}

\section{DERIVATION OF EQUation 6}

Consider a tagged node in Cell- $i$. Let

$A_{i}(T)$ : number of attempts made by the tagged node up to time $T$

$C_{i}(T)$ : number of collisions as seen by the tagged node up to time $T$

$A_{i}^{\mathcal{A}}(T)$ : number of attempts made by the node in State- $\mathcal{A}$ up to time $T$

$C_{i}^{\mathcal{A}}(T)$ : number of collisions as seen by the tagged node in State- $\mathcal{A}$ up to time $T$

$B_{i}^{\mathcal{A}}(T)$ : number of back-off slots in Cell- $i$ in State- $\mathcal{A}$ up to time $T$

Then, we have

$$
\begin{aligned}
& \gamma_{i}=\lim _{T \longrightarrow \infty} \frac{C_{i}(T)}{A_{i}(T)}=\frac{\lim _{T \longrightarrow \infty} \frac{1}{T} \sum_{T \rightarrow \mathcal{A}_{i \in \mathcal{U}_{\mathcal{A}}}} C_{i}^{\mathcal{A}}(T)}{\lim _{\mathcal{A}} \frac{1}{T} \sum_{\mathcal{A} \in \mathcal{\mathcal { A }}: i \in \mathcal{U}_{\mathcal{A}}} A_{i}^{\mathcal{A}}(T)}=\frac{\lim _{T \rightarrow \infty} \frac{1}{T} \sum_{\mathcal{A} \in \mathcal{A}: i \in \mathcal{U}_{\mathcal{A}}} B_{i}^{\mathcal{A}}(T) \times \frac{C_{i}^{\mathcal{A}}(T)}{B_{i}^{\mathcal{A}}(T)}}{\lim _{T \rightarrow \infty} \frac{1}{T} \sum_{\mathcal{A} \in \mathcal{A}: i \in \mathcal{U}_{\mathcal{A}}} B_{i}^{\mathcal{A}}(T) \times \frac{A_{i}^{\mathcal{A}}(T)}{B_{i}^{\mathcal{A}}(T)}} \\
& =\frac{\sum_{\mathcal{A} \in \mathcal{A}: i \in \mathcal{U}_{\mathcal{A}}}\left(\lim _{T \longrightarrow \infty} \frac{B_{i}^{\mathcal{A}}(T)}{T}\right) \times\left(\lim _{T \longrightarrow \infty} \frac{C_{i}^{\mathcal{A}}(T)}{B_{i}^{\mathcal{A}}(T)}\right)}{\sum_{\mathcal{A} \in \mathcal{A}: i \in \mathcal{U}_{\mathcal{A}}}\left(\lim _{T \longrightarrow \infty} \frac{B_{i}^{\mathcal{A}}(T)}{T}\right) \times\left(\lim _{T \longrightarrow \infty} \frac{A_{i}^{\mathcal{A}}(T)}{B_{i}^{\mathcal{A}}(T)}\right)} \\
& =\frac{\sum_{\mathcal{A} \in \mathcal{A}: i \in \mathcal{U}_{\mathcal{A}}}\left(\lim _{T \longrightarrow \infty} \frac{B_{i}^{\mathcal{A}}(T)}{T}\right) \times\left(\lim _{T \longrightarrow \infty} \frac{A_{i}^{\mathcal{A}}(T)}{B_{i}^{\mathcal{A}}(T)}\right) \times\left(\lim _{T \rightarrow \infty} \frac{C_{i}^{\mathcal{A}}(T)}{A_{i}^{\mathcal{A}}(T)}\right)}{\sum_{\mathcal{A} \in \mathcal{A}: i \in \mathcal{U}_{\mathcal{A}}}\left(\lim _{T \longrightarrow \infty} \frac{B_{i}^{\mathcal{A}}(T)}{T}\right) \times\left(\lim _{T \longrightarrow \infty} \frac{A_{i}^{\mathcal{A}}(T)}{B_{i}^{\mathcal{A}}(T)}\right)} \\
& =\frac{\sum_{\mathcal{A} \in \mathcal{A}: i \in \mathcal{U}_{\mathcal{A}}}\left(\frac{\pi(\mathcal{A})}{\sigma}\right) \times \beta_{i} \times\left(1-\left(1-\beta_{i}\right)^{n_{i}-1} \prod_{j \in \mathcal{N}_{i}: j \in \mathcal{U}_{\mathcal{A}}}\left(1-\beta_{j}\right)^{n_{j}}\right)}{\sum_{\mathcal{A} \in \mathcal{A}: i \in \mathcal{U}_{\mathcal{A}}}\left(\frac{\pi(\mathcal{A})}{\sigma}\right) \times \beta_{i}} \\
& =\frac{\sum_{\mathcal{A} \in \mathcal{A}: i \in \mathcal{U}_{\mathcal{A}}} \pi(\mathcal{A})\left(1-\left(1-\beta_{i}\right)^{n_{i}-1} \prod_{j \in \mathcal{N}_{i}: j \in \mathcal{U}_{\mathcal{A}}}\left(1-\beta_{j}\right)^{n_{j}}\right)}{\sum_{\mathcal{A} \in \mathcal{A}: i \in \mathcal{U}_{\mathcal{A}}} \pi(\mathcal{A})}
\end{aligned}
$$

where the last but one step follows from the facts that: (i) the time spent in State- $\mathcal{A}$ up to time $T$ is equal to $\pi(\mathcal{A}) T$ and if Cell- $i$ is in back-off in State- $\mathcal{A}$, then $B_{i}^{\mathcal{A}}(T)=\frac{\pi(\mathcal{A}) T}{\sigma}$, (ii) the second limit within the brackets in the numerator is the long-run fraction of back-off slots in State- $\mathcal{A}$ in which the tagged node attempts which is equal to $\beta_{i}$ irrespective of State- $\mathcal{A}$ given that Cell- $i$ is in back-off in State- $\mathcal{A}$, and (iii) the third limit within brackets in the numerator is the long-run fraction of attempts made by the tagged node in State- $\mathcal{A}$ that result in collisions and depends on the set of neighbors that are also in back-off in State- $\mathcal{A}$. 
APPENDIX B

PROOF OF THEOREM 5.1

Solving the balance equations for the CTMC $\{\mathcal{A}(t), t \geq 0\}$ (Equation 4) together with the normalization equation, we obtain

$$
\pi(\Phi)=\frac{1}{\sum_{\mathcal{A} \in \mathcal{A}}\left(\prod_{i \in \mathcal{A}} \rho_{i}\right)}=\frac{1}{\Delta}
$$

Equation 7 can now be expanded as

$$
\begin{aligned}
& x_{i}=\sum_{\mathcal{A} \in \mathcal{A}: i \in \mathcal{A}} \pi(\mathcal{A})+\sum_{\mathcal{A} \in \mathcal{A}_{i \in \mathcal{U}_{\mathcal{A}}}} \pi(\mathcal{A}) \quad\left(\text { since } \mathcal{A} \cap \mathcal{U}_{\mathcal{A}}=\Phi\right) \\
& =\sum_{\mathcal{A} \in \mathcal{A}: i \in \mathcal{A}}\left(\prod_{j \in \mathcal{A}} \rho_{j}\right) \pi(\Phi)+\sum_{\mathcal{A} \in \mathcal{A}: i \in \mathcal{U}_{\mathcal{A}}}\left(\prod_{j \in \mathcal{A}} \rho_{j}\right) \pi(\Phi) \quad \text { (by Equation 4) } \\
& =\frac{1}{\Delta}\left[\sum_{\mathcal{A} \in \mathcal{A}: i \in \mathcal{A}}\left(\prod_{j \in \mathcal{A}} \rho_{j}\right)+\sum_{\mathcal{A} \in \mathcal{A}: i \in \mathcal{U}_{\mathcal{A}}}\left(\prod_{j \in \mathcal{A}} \rho_{j}\right)\right] \quad \text { (by Equation 15) }
\end{aligned}
$$

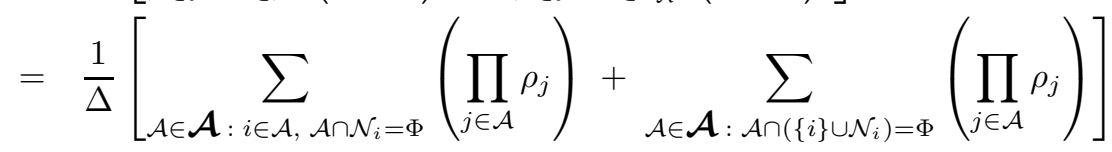

$$
\begin{aligned}
& =\frac{1}{\Delta}\left[\rho_{i} \sum_{\mathcal{A} \in \mathcal{A}: \mathcal{A} \cap\{i\}=\Phi,{\mathcal{A} \cap \mathcal{N}_{i}=\Phi}_{j \in \mathcal{A}}}\left(\prod_{j \in} \rho_{j}\right)+\sum_{\mathcal{A} \in \mathcal{A}: \mathcal{A} \cap\left(\{i\} \cup \mathcal{N}_{i}\right)=\Phi}\left(\prod_{j \in \mathcal{A}} \rho_{j}\right)\right],
\end{aligned}
$$

where the fourth step follows from the facts that: (i) if Cell- $i$ is active, then none of its neighbors can be active, and (i) if Cell- $i$ is in back-off, then neither Cell- $i$ nor any of its neighbors can be active. Observe now that, the independent sets of $\mathcal{G}_{i}$ are also independent sets of $\mathcal{G}$. Thus, $\Delta_{i}$ can be obtained by restricting the summation in Equation 8 to only those independent sets of $\mathcal{G}$ that do not contain Cell- $i$ or any cell in $\mathcal{N}_{i}$, i.e.,

$$
\Delta_{i}:=\sum_{\mathcal{A} \in \mathcal{A}: \mathcal{A} \cap\left(\{i\} \cup \mathcal{N}_{i}\right)=\Phi}\left(\prod_{i \in \mathcal{A}} \rho_{i}\right)=\sum_{\mathcal{A} \in \mathcal{A}: \mathcal{A} \cap\{i\}=\Phi \text { and }{\mathcal{A} \cap \mathcal{N}_{i}=\Phi}_{i \in \mathcal{A}}}\left(\prod_{i} \rho_{i}\right)
$$

where the second equality follows from the fact that union of two sets will be equal to the empty set iff each of the sets is an empty set. Applying Equation 17 in the last step of Equation 16 , we obtain Equation 9. 